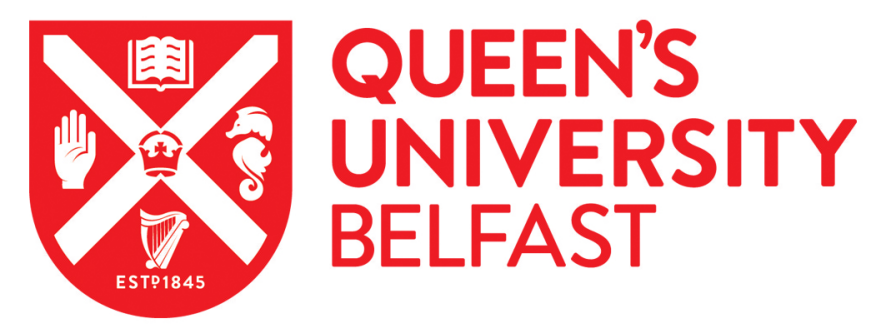

\title{
On the Complexity of Universal Leader Election
}

Kutten, S., Pandurangan, G., Peleg, D., Robinson, P., \& Trehan, A. (2015). On the Complexity of Universal Leader Election. Journal of the ACM, 62(1), [7]. https://doi.org/10.1145/2699440

Published in:

Journal of the ACM

Document Version:

Peer reviewed version

Queen's University Belfast - Research Portal:

Link to publication record in Queen's University Belfast Research Portal

Publisher rights

(c) ACM, 2015. This is the author's version of the work. It is posted here by permission of ACM for your personal use. Not for redistribution. The definitive version was published in

ACM Journal, Vol 62, Issue 1, Feb 2015. http://doi.acm.org/10.1145/10.1145/2699440

\section{General rights}

Copyright for the publications made accessible via the Queen's University Belfast Research Portal is retained by the author(s) and / or other copyright owners and it is a condition of accessing these publications that users recognise and abide by the legal requirements associated with these rights.

Take down policy

The Research Portal is Queen's institutional repository that provides access to Queen's research output. Every effort has been made to ensure that content in the Research Portal does not infringe any person's rights, or applicable UK laws. If you discover content in the Research Portal that you believe breaches copyright or violates any law, please contact openaccess@qub.ac.uk. 


\title{
On the Complexity of Universal Leader Election*
}

\author{
Shay Kutten ${ }^{\dagger} \quad$ Gopal Pandurangan ${ }^{\ddagger}$ David Peleg ${ }^{\S} \quad$ Peter Robinson ${ }^{\circledR}$ \\ Amitabh Trehan $\|$
}

December 2, 2015

\begin{abstract}
Electing a leader is a fundamental task in distributed computing. In its implicit version, only the leader must know who is the elected leader. This paper focuses on studying the message and time complexity of randomized implicit leader election in synchronous distributed networks. Surprisingly, the most "obvious" complexity bounds have not been proven for randomized algorithms. In particular, the seemingly obvious lower bounds of $\Omega(m)$ messages, where $m$ is the number of edges in the network, and $\Omega(D)$ time, where $D$ is the network diameter, are non-trivial to show for randomized (Monte Carlo) algorithms. (Recent results, showing that even $\Omega(n)$, where $n$ is the number of nodes in the network, is not a lower bound on the messages in complete networks, make the above bounds somewhat less obvious). To the best of our knowledge, these basic lower bounds have not been established even for deterministic algorithms, except for the restricted case of comparison algorithms, where it was also required that nodes may not wake up spontaneously and that $D$ and $n$ were not known. We establish these fundamental lower bounds in this paper for the general case, even for randomized Monte Carlo algorithms. Our lower bounds are universal in the sense that they hold for all universal algorithms (namely, algorithms that work for all graphs), apply to every $D, m$, and $n$, and hold even if $D, m$, and $n$ are known, all the nodes wake up simultaneously, and the algorithms can make any use of node's identities. To show that these bounds are tight, we present an $O(m)$ messages algorithm. An $O(D)$ time leader election algorithm is known. A slight adaptation of
\end{abstract}

\footnotetext{
*A preliminary version of this article appeared in the proceedings of the 32nd ACM Symposium on Principles of Distributed Computing (PODC) 2013, pages 100-109. Shay Kutten and Amitabh Trehan were supported in part by the Israel Science Foundation and by the Technion TASP center. Gopal Pandurangan was supported in part by the following grants: Nanyang Technological University grant M58110000, Singapore Ministry of Education (MOE) Academic Research Fund (AcRF) Tier 2 grant MOE2010-T2-2-082, Singapore MOE AcRF Tier 1 grant MOE2012T1-001-094, and a grant from the US-Israel Binational Science Foundation grant 2008348. Work done when the author was affiliated with the Division of Mathematical Sciences, Nanyang Technological University \& Department of Computer Science, Brown University. David Peleg was supported in part by the Israel Science Foundation (grant 894/09), the United States-Israel Binational Science Foundation (grant 2008348), the Israel Ministry of Science and Technology (infrastructures grant), the I-CORE program of the Israel PBC and ISF (grant 4/11), and the Citi Foundation. Peter Robinson was supported in part by the following grants: Nanyang Technological University grant M58110000, Singapore MoE Academic Research Fund (AcRF) Tier 2 grant MOE2010-T2-2-082, Fault-tolerant Communication Complexity in Wireless Networks from the Singapore MoE AcRF-2.

${ }^{\dagger}$ Faculty of IE\&M, Technion

${ }^{\ddagger}$ Department of Computer Science, University of Houston

${ }^{\S}$ Department of Computer Science, The Weizmann Institute

`Department of Computer Science, National University of Singapore

"High Performance \& Distributed Computing, EEECS, Queen's University Belfast
} 
our lower bound technique gives rise to an $\Omega(m)$ message lower bound for randomized broadcast algorithms.

An interesting fundamental problem is whether both upper bounds (messages and time) can be reached simultaneously in the randomized setting for all graphs. The answer is known to be negative in the deterministic setting. We answer this problem partially by presenting a randomized algorithm that matches both complexities in some cases. This already separates (for some cases) randomized algorithms from deterministic ones. As first steps towards the general case, we present several universal leader election algorithms with bounds that trade-off messages versus time. We view our results as a step towards understanding the complexity of universal leader election in distributed networks.

\section{Introduction}

Leader election is a fundamental and classical problem in distributed computing. Due to shortage of space, we rely on the reader's familiarity with its long history and many theoretical implications. Previous work is too rich to survey here, see, e.g., [3, 16, 22, 23]. Still, let us stress that this long-studied task is relevant today more than ever, with its practical applications to the emerging area of large scale and resource-constrained networks such as peer-to-peer networks (e.g., that of Akamai [19]), ad hoc and sensor networks (e.g., [10, 24]). For example, minimizing messages and time for basic tasks such as leader election can help in minimizing energy consumption in ad hoc and sensor networks. Hence, it is desirable to achieve fast, low cost and scalable leader election. This is one of the reasons why this paper concentrates on randomized algorithms, that have been shown to reduce complexity dramatically in various contexts. (In fact, it was recently shown that the randomized message complexity of leader election in complete graphs is sublinear, $O\left(\sqrt{n} \log ^{3 / 2} n\right)$ [14], where $n$ is the number of nodes.) Interestingly, although the leader election task is so well studied, some basic theoretical questions concerning its complexity have not been answered yet, especially (but not only) for the randomized case.

Informally, the leader election task requires a group of processors in a distributed network to elect a unique leader among themselves, i.e., exactly one processor must output the decision that it is the leader, say, by changing a special status component of its state to the value leader, with all the other nodes changing their status component to the value non-leader. These nodes need not be aware of the identity of the leader. This implicit version of leader election is rather standard (cf. [16]), and is sufficient in many applications, e.g., its original application for token generation in a token ring environment [15]. (In the explicit variant, every node must also know the identity of the unique leader.) This paper focuses on implicit leader election, although our algorithms apply to the explicit version as well.

The study of leader election algorithms is usually concerned with both message and time complexity. Both may appear to be very well understood. For example, Awerbuch [4] presented an $O(n)$ rounds, $O(m+n \log n)$ messages deterministic algorithm that was claimed to be optimal both in terms of time complexity and in terms of message complexity. As demonstrated in [20], the time in Awerbuch's algorithm may be optimal only existentially, that is, only in the case that $n=O(D)$, where $D$ the diameter of the graph. Moreover, these claims of optimality rely on the tacit assumption that $D$ and $m$ (the number of edges) are lower bounds on the time and the number of messages required for leader election, respectively. Surprisingly, even for deterministic algorithms, the only proof we are aware of for a lower bound of $\Omega(m)$ on the message complexity of leader election is for the rather restricted case where (a) the algorithms are only comparison algorithms (that may not 
manipulate the actual value of node's identities, but only compare identities with each other), (b) spontaneous wakeup of the nodes is not guaranteed, and (c) network parameters (such as $n$ ) are not known to the nodes. This restricted case admits a very short and elegant proof, cf. [23]. Unfortunately, that proof fails completely if one of the assumptions is removed ${ }^{1}$. (As a by product of our results for randomized algorithms, we get rid of all these special assumptions for deterministic algorithms too.) For the time complexity of leader election, the situation is even less stable, and the lower bound of $D$ seems to be folklore, cf. [5, 23].)

Let us assume for a moment that the above lower bounds were indeed "obvious" (though not formally proven for the general case) for deterministic algorithms. Are they indeed that obvious for randomized ones? The work of [14] demonstrated that, for randomized algorithms, the seemingly obvious lower bound of $\Omega(n)$ messages for a complete graph (as well as other classes of graphs with sufficiently small mixing times such as expanders and hypercubes) does not hold. Specifically, it presented an algorithm that executes in $O(1)$ time and uses only $O\left(\sqrt{n} \log ^{3 / 2} n\right)$ messages to elect a leader in a complete graph (and similarly for other families of high-expansion graphs). Consequently, it would appear that the obvious lower bounds on time and messages must be revisited, especially for randomized algorithms.

This paper concerns universal leader election algorithms, namely, algorithms that work for all graphs. The unconditional randomized lower bounds of $\Omega(m)$ messages and $\Omega(D)$ time shown in this paper (cf. Table 1) subsume the above deterministic bounds in a general way. These bounds apply to a large class of graphs for (essentially) every given $m, D$, and $n$. They hold even for non-comparison algorithms and even if nodes have knowledge of these parameters. They also hold for synchronous networks, and even if all the nodes wake up simultaneously. Finally, they hold not only for the $\mathcal{C O N G \mathcal { S }} \mathcal{S}$ model [21], where sending a message of $O(\log n)$ bits takes one unit of time, but also for the $\mathcal{L O C} \mathcal{A L}$ model, where the number of bits in a message is allowed to be arbitrary.

We note that the universal lower bounds of $\Omega(m)$ and $\Omega(D)$ do not follow from currently known results. There are several known lower bounds for deterministic leader election algorithms in cycles (e.g., [8]) and complete graphs (e.g., [13, 2]), which also imply bounds for (deterministic) universal algorithms. These results alone do not, however, imply that a universal algorithm cannot do significantly better in other classes of networks. For example, the deterministic lower bound of $\Omega(n \log n)$ messages in ring graphs (cf. $[8,23]$ ) does not imply a lower bound of $\Omega(m)$ messages (even for deterministic algorithms) in general graphs. Moreover, it does not even imply the necessity of $\Omega(n \log n)$ messages for every graph, since there exist graphs with $n$ nodes where the number of messages required is smaller (e.g., a star graph). It may even be possible to design a universal election algorithm that will use only $O(n)$ messages when executed on a star graph.

Compared to deterministic algorithms, lower bounds (and their proofs) for randomized algorithms, particularly Monte Carlo ones, are more delicate. For example, consider the following simple algorithm (which assumes the nodes know $n$ ): "Each node elects itself as leader with probability $1 / n$." The probability of this algorithm resulting in exactly one leader is $\left(\begin{array}{l}n \\ 1\end{array}\right) \frac{1}{n}(1-1 / n)^{n-1} \approx 1 / e \approx$

\footnotetext{
${ }^{1}$ Interestingly, even in the explicit deterministic variant, an "obvious" lower bound of $\Omega(m)$ messages was not known. Indeed, the explicit version seems to require a broadcast of the leader's name. Still, the known lower bound of $\Omega(m)$ on broadcast was shown only for time-bounded deterministic algorithms that use messages of bounded size [5]. Hence in the general case, it was not known that conveying the leader's identity to every node consumes $\Omega(m)$ messages. As opposed to the number of edges, still for the explicit variant, $\Omega(n)$ (the number of nodes) and $\Omega(D)$ do seem to be known lower bounds on the messages and time. Nevertheless, $\Omega(D)$ time is not obvious for the implicit variant studied here.
} 
0.368. Hence there exists a randomized algorithm that elects a leader in one time unit, without sending any messages, and succeeds with constant (albeit small) probability! In contrast, as proved later in the paper, if the success probability is required to be a somewhat larger constant, then the time lower bound becomes $\Omega(D)$ and the message lower bound becomes $\Omega(m)$.

To the best of our knowledge, previous work on time complexity bounds for leader election in general networks is scarce. In a recent work [9], the authors study deterministic algorithms for variants of leader election in anonymous networks called weak (resp., strong) leader election, which require the algorithm to elect a leader in the given network if possible. It is shown therein that $D+\lambda$ is a lower bound in this setting, where $\lambda$ is a symmetry parameter, which is the smallest depth at which the views of the nodes become distinguishable.

Over two decades ago, the following basic open problem was raised in [20]: Is it possible to design a universal algorithm for leader election that is simultaneously both time and message optimal? In view of the lower bounds in this paper, this question can be reformulated as follows: Is there an $O(D)$ time and $O(m)$ messages universal leader election algorithm? The answer is negative if we restrict ourselves to deterministic algorithms, since it is known that for a cycle any $O(n)$ time deterministic algorithm requires at least $\Omega(n \log n)$ messages (even when nodes know $n)$ [8]. However, the problem still stands for randomized algorithms. We provide a partial answer for this problem by presenting a randomized algorithm that matches both complexities for $m \geqslant n^{1+\varepsilon}$ (for any fixed constant $\varepsilon>0$ ), assuming $n$ is known. This already separates randomized algorithms from deterministic ones. Another such case (for every $m$ ) when both lower bounds can be matched is when $n$ and $D$ are known. As first steps for the more general case, we present several universal leader election algorithms with bounds that trade-off messages versus time. In particular, to also show that our lower bounds are tight, we present a simple deterministic algorithm that uses $O(m)$ messages. An $O(D)$ time algorithm is already known [20].

\subsection{Our Results}

This paper presents lower and upper bounds for universal leader election algorithms in synchronous arbitrary networks. Our results on leader election are summarized in Table 1. The tight bounds are the lower ones - Theorem 3.13 and Theorem 3.1, as demonstrated by Theorem 4.1 (and [20]). Corollary 3.12 shows that a slight adaptation of our lower bound technique implies an $\Omega(m)$ message lower bound for solving the broadcast problem. Note that Theorem 4.4.(B) presents a case where one can match both lower bounds simultaneously with a constant (though close to 1) probability. Corollary 4.2 shows a case where both bounds can be matched with high success probability ${ }^{2}$ (but with a constraint on $m$ ). Corollary 4.6 demonstrates a case with probability 1 , but with an extra assumption (knowledge of $D$ ). The other results in the table may be of interest by themselves. They were obtained on the way to reaching the above results, or in trying to get close to a tight upper bound for the general case. We next elaborate on our bounds and techniques used.

Lower Bounds: As mentioned earlier, to the best of our knowledge, lower bounds for randomized (especially Monte Carlo) algorithms have not been studied. Here we present two basic lower bounds that apply to randomized algorithms, including Monte Carlo algorithms with (suitably large) constant success probability: an $\Omega(m)$ bound on the number of messages and an $\Omega(D)$ bound on the time. Our message lower bound (cf. Theorem 3.1) applies to any $m$ and $n$, i.e.,

\footnotetext{
${ }^{2}$ Throughout, "with high probability (w.h.p.)" means with probability at least $1-1 / n$.
} 
we show that given any $n$ and $m$, there exists a graph with $\Theta(n)$ nodes and $\Theta(m)$ edges, where the lower bound holds. Our time lower bound (cf. Theorem 3.13) states that for every $n$ and $D$ $(2<D<n)$, there exists a graph with $\Theta(n)$ nodes and $\Theta(D)$ diameter for which the time needed is $\Omega(D)$, even with constant success probability. Also, these lower bounds hold even if the nodes have knowledge of the global parameters $n, D$ and $m$. Our lower bounds apply to all algorithms (and not just comparison-based ones) and hold even if the all nodes wake up simultaneously.

Our message lower bound is proved by first showing a lower bound for a related problem referred to as "bridge crossing $(B C)$ ". In the bridge crossing problem, it is required that at least one message is sent across a "bridge" edge connecting two specific subgraphs. We then show conditions under which any universal leader election algorithm must solve BC. We first show a lower bound of the expected message complexity of deterministic algorithms for BC and LE, and then use Yao's lemma (cf. Lemma 3.2) to show that it applies also to the expected message complexity of randomized algorithms on the worst-case input. The proof involves constructing a suitable graph that ensures that a knowledge of $n, m, D$, and identity assignment will be useless to any algorithm; it also involves a counting argument to lower bound the number of messages sent.

Our $\Omega(D)$ time lower bound is proven directly for Monte Carlo algorithms by a probabilistic argument. We show that for a suitably constructed graph of a given size and diameter, any Monte Carlo algorithm that needs to succeed with a suitably large constant probability must communicate over a distance of $\Omega(D)$ edges. Otherwise, there might not be a unique leader.

Upper Bounds: The purpose of algorithms in this paper is twofold:

(1) to show the tightness of the message lower bound (the tightness of the time lower bound follows from [20]), and

(2) addressing the fundamental question: can both lower bounds $O(D)$ time and $O(m)$ messages be matched or approached simultaneously?

For goal (1) above, we show that there exists a deterministic universal algorithm that is optimal in the number of messages, i.e., an $O(m)$ algorithm. (Cf. Theorem 4.1). However, this algorithm takes arbitrary (albeit finite) time (which depends exponentially on the size of the smallest ID). This algorithm is a generalization of the one presented by Fredrickson and Lynch for rings [8].

We now highlight the techniques behind the universal algorithms for goal (2), and point at their

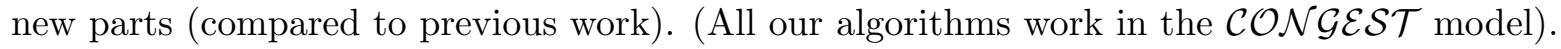

\subsubsection{Matching (sometimes) both lower bounds simultaneously}

As mentioned earlier, only randomized algorithms have the hope of matching both lower bounds simultaneously. We start with the least element lists (Least-El list) algorithm of [11]. It can be used to elect a unique leader (with probability 1) in $O(D)$ time and $O(m \min (\log n, D)$ ) messages [11]. The main idea was to use random IDs. Each node simply floods its ID and the largest ID wins. If the IDs are chosen randomly, it can be shown that the number of messages that every node $v$ has to forward is bounded by $O(\log n) \operatorname{deg}(v)$, where $\operatorname{deg}(v)$ is $v$ 's degree; this yields the desired message bound. However, in previous work, the resulting algorithm needed to know $n$, the number of nodes.

In the current paper, we first show that a standard technique can be used to get rid of this assumption, by showing that nodes can get an estimate of $\log n$ (up to a constant factor) in $O(m \min (\log n, D))$ messages and $O(D)$ time: While this yields a Las Vegas randomized algorithm (cf. Corollary 4.5), its complexity still falls short of matching both lower bounds. Next, we 
present another improvement to the Least-El list algorithm of [11], showing that if, instead of allowing every node to be a candidate, only $\log n$ nodes are allowed to be candidates, then the (expected) message complexity can be improved to $O(m \log \log n)$ with the same time bound. (Note that the candidates are chosen randomly and do not have any a priori knowledge of each other.) This yields a Monte-Carlo algorithm that succeeds with high probability. More generally, we show it is possible to obtain a trade-off between the success probability and the number of messages. In particular, we get an $O(m)$ messages, $O(D)$ time algorithm with large constant success probability (for any large pre-specified constant). See Theorem 4.4.(B). This matches our lower bounds of time and messages for the case when the success probability is constant. However, the above improvement requires nodes to have knowledge of $n$.

We also manage to match both lower bounds simultaneously for graphs that are "somewhat dense," i.e., with $m>n^{1+\varepsilon}$ for any fixed constant $\varepsilon>0$ (known to the algorithm). This is done by combining a randomized spanner algorithm due to [6] and the above Least-El list algorithm to achieve leader election with high probability. See Corollary 4.2.

Finally, we show in Corollary 4.6 that if nodes have knowledge of both $n$ and $D$, then one can obtain a randomized Las Vegas algorithm with expected time complexity $O(D)$ and expected message complexity $O(m)$.

\subsubsection{Approaching both lower bounds simultaneously}

The Least-El list based algorithms above indeed match both lower bounds sometimes. However, at some other times their message complexity is higher than $m$ by a multiplicative factor that may be larger than a constant. For completeness, we also present a randomized algorithm with a better message complexity in the worst case, although at some small time penalty. See Theorem 4.7. This Monte-Carlo algorithm, referred to as the "clustering algorithm", is not based on the Least-El list approach, used by the other randomized algorithms above. In this algorithm, which also relies on prior knowledge of $n$, about $\log n$ nodes choose to be candidates and grow clusters till they essentially meet. The approach then is to first sparsify this graph and reduce the number of edges to about $\min \left(m, n+\log ^{2} n\right)$. However, this sparsification increases the diameter of the residual graph to $O(D \log n)$. One can then apply the Least-El list algorithm to the residual graph to obtain an overall bound of $O(D \log n)$ time and $O(m+n \log n)$ messages.

Finally, as mentioned, in the deterministic case, it is impossible to match both lower bounds simultaneously. Hence, [1] posed that goal of reaching $O(m+n \log n)$ messages and $O(D)$ time simultaneously. They presented a sketch of an elegant deterministic algorithm that "grows kingdoms"; a leader candidate's kingdoms keep growing (by building BFS trees) till only one kingdom is left. Unfortunately, one can show counter examples to the hope that this algorithm doubles the diameters of winning kingdoms every round. We present a modified variant of the algorithm of [1]. The modification ensures that the upper bound on the number of kingdoms is reduced by a constant factor in every phase. Our algorithm requires no knowledge of $n$ or any other parameter (it does however require unique identities, which is necessary.) This yields a $O(D \log n)$ time and $O(m \log n)$ messages deterministic algorithm. It is an open question whether the running time can be improved to $O(D)$, with the same number of messages, in the deterministic case. 


\begin{tabular}{lllll}
\hline & Time & Messages & Knowledge & Success Probability \\
\hline Lower Bounds: & & & & \\
Theorem 3.1 & - & $\Omega(m)^{\dagger}$ & $n, m, D$ & $\geqslant 53 / 56$ \\
Theorem 3.13 & $\Omega(D)^{*}$ & - & $n, m, D$ & $\geqslant \frac{15}{16}\left(1+n^{-2}\right)$ \\
\hline Randomized Algorithms: & & & \\
Theorem 4.4 & $O(D)$ & $O(m \min (\log f(n), D))^{\dagger, \#}$ & $n$ & $\geqslant 1-1 / e^{\Theta(f(n))}$ \\
Theorem 4.4.(A) & $O(D)$ & $O(m \min (\log \log n, D))^{\dagger}$ & $n$ & $\geqslant 1-n^{-1}$ \\
Theorem 4.4.(B) & $O(D)$ & $O(m)^{\dagger}$ & $n$ & $\geqslant 1-\varepsilon^{\dagger \dagger}$ \\
Corollary 4.2 & $O(D)$ & $O(m)^{\dagger}$ if $m \geqslant n^{1+\varepsilon} \dagger \dagger$ & $n$ & $\geqslant 1-n^{-1}$ \\
Corollary 4.5 & $O(D)$ & $O(m \min (\log n, D))^{\$}$ & - & 1 \\
Corollary 4.6 & $O(D)^{\dagger}$ & $O(m)^{\dagger}$ & $n, D$ & 1 \\
Theorem 4.7 & $O(D \log n)^{\$}$ & $O(m+n \log n)^{\$}$ & $n$ & $\geqslant 1-n^{-1}$ \\
\hline
\end{tabular}

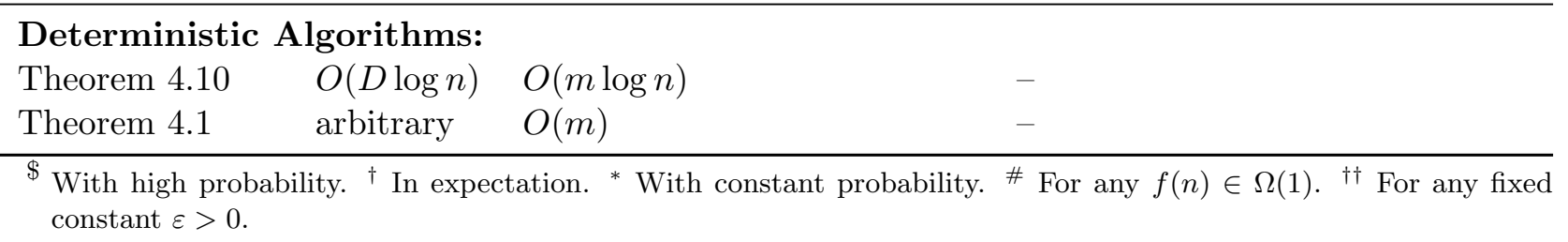

Table 1: Upper and lower bounds for universal leader election algorithms.

\section{Preliminaries}

We consider a system of $n$ nodes, represented as an undirected connected (not necessarily complete) graph $G=(V, E)$. Each node $u$ runs an instance of a distributed algorithm and has a unique identifier $\mathrm{ID}_{u}$ of $O(\log n)$ bits chosen by an adversary from an arbitrary set of integers $Z$ of size $n^{4}$ (the nodes themselves may not have knowledge of $n$, nor of $Z$ ). The lower bounds hold even when nodes have unique identities (IDs). However, some of our algorithms do not require that assumption. Hence, the randomized algorithms in this paper also apply for anonymous networks. To make the lower bounds more general, we assume that all nodes wake up simultaneously at the beginning of the execution.

The computation advances in synchronous rounds, where in every round, nodes can send messages, receive messages that were sent in the same round by neighbors in $G$, and perform some

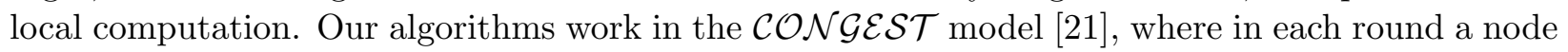
can send at most one message of size $O(\log n)$ bits on a single edge. In contrast, our lower bounds apply even in the $\mathcal{L O C} \mathcal{A} \mathcal{L}$ model [21], where there is no restriction on message size.

For randomized (Las Vegas and Monte Carlo) algorithms, we also assume that every node has access to the outcome of unbiased private coin flips. Messages are the only means of communication; in particular, nodes cannot access the coin flips of other nodes, and do not share any memory. The classical leader election literature distinguishes between the simultaneous wakeup model where all nodes are awake initially and start executing the algorithm simultaneously, and the adversarial wakeup model where the nodes are awoken at arbitrary points in time, with the restriction that nodes wake upon receiving a message and at least one node is initially awake. Our lower bounds hold even if the nodes are initially awake. In contrast, the analysis of some of the algorithms holds even for the case of adversarial wakeup. 
Initially, each node is given a port numbering where each port is connected to an incident edge leading to a neighbor. However, the node has no knowledge of the neighbor at the other endpoint of edge. Recall that our lower bounds hold even if the nodes know some of the graph parameters, such as $n, D$, and $m$. Some of our algorithms work without this assumption. However, other algorithms rely on the assumption that the nodes know one or more of these parameters (see Table 1).

We now define the leader election problem formally. Every node $u$ has a special variable status $u$ that can be set to a value in $\{\perp$, NON-ELECTED, ELECTED $\}$; initially status $\boldsymbol{s}_{u}=\perp$. An algorithm $A$ solves leader election in $T$ rounds if, from round $T$ on, exactly one node has its status set to ELECTED while all other nodes are in state NON-ELECTED.

We say that $A$ is a universal leader election algorithm, with error probability $\varepsilon$, if for any choice of $n$ and $m$, the probability that $A$ succeeds is at least $1-\varepsilon$ on any network of $n$ nodes and $m$ edges, and any ID assignment chosen from any integer set of large polynomial (in $n$ ) size; for our lower bounds we assume that $|Z| \geqslant n^{4}$. In particular, if $A$ is a deterministic algorithm or a randomized Las Vegas algorithm, then $A$ is universal if and only if it achieves leader election on every network under all ID assignments.

\section{Randomized Lower Bounds}

\subsection{Message Complexity Lower Bound}

Theorem 3.1. Let $R$ be a universal leader election algorithm that succeeds with probability at least $1-\beta$, for some constant $\beta \leqslant 3 / 56$. For every sufficiently large $n$ and $n \leqslant m \leqslant\left(\begin{array}{l}n \\ 2\end{array}\right)$, there exists a (connected) graph $G$ of $n$ nodes and $\Theta(m)$ edges, such that the expected number of messages used by $R$ on $G$ is $\Omega(m)$. The above holds even if $n, m$ and $D$ are known to the algorithm and all nodes wake up simultaneously.

For simplicity, we first describe the proof assuming the nodes do not know the diameter $D$. (More explicitly, we establish a weaker version of the theorem, dealing only with strong algorithms, which succeed even when the nodes do not know D.) At the end of the proof, we explain why this proof fails for weaker algorithms, which are guaranteed to work correctly only when the nodes know $D$, and then outline the modifications necessary to allow the proof to handle this harder case as well.

Construction of Dumbbell Graphs The proof is based on constructing a graph family referred to as dumbbell graphs. Given $R, n$ and $m$, pick one specific 2-connected graph $G_{0}$ of $n$ nodes and $m$ edges, and a range $Z=\left[1, n^{4}\right]$ of ID's. This $G_{0}$ has many instantiations, obtained by fixing the node ID assignment and the port number mapping. An ID assignment is a function $\varphi: V\left(G_{0}\right) \mapsto Z$. A port mapping for node $v$ is a mapping $P_{v}:\left[1, d e g_{v}\right] \mapsto \Gamma(v)$ (namely, $v$ 's neighbors). A port mapping for the graph $G_{0}$ is $P=\left\langle P_{v_{1}}, \ldots, P_{v_{n}}\right\rangle$. Every choice of $\varphi$ and $P$ yields a concrete graph $G_{\varphi, P}$. Denote the set of id's of this graph by $I D\left(G_{\varphi, P}\right)=\left\{\varphi(v) \mid v \in V\left(G_{0}\right)\right\}$. Let $\mathcal{G}$ be the collection of concrete graphs $G_{\varphi, P}$ obtained from $G_{0}$. For a graph $G \in \mathcal{G}$, and an edge $e$ of $G_{0}$, the "open graph" $G[e]$ is obtained by erasing $e$ and leaving the two ports that were attached to it empty. Let $\mathcal{G}^{\text {open }}$ be the collection of open graphs obtained from $G_{0}$.

For two open graphs $G^{\prime}\left[e^{\prime}\right]$ and $G^{\prime \prime}\left[e^{\prime \prime}\right]$ with disjoint sets of ID's, $I D\left(G^{\prime}\left[e^{\prime}\right]\right) \cap I D\left(G^{\prime \prime}\left[e^{\prime \prime}\right]\right)=\emptyset$, let Dumbbell $\left(G^{\prime}\left[e^{\prime}\right], G^{\prime \prime}\left[e^{\prime \prime}\right]\right)$ be the graph obtained by taking one copy of each of these graphs, and connecting their open ports. Hence a dumbbell graph is composed of two open graphs plus two 
connecting edges, referred to as bridges. Moreover, we say that $G^{\prime}\left[e^{\prime}\right]$ participates on the left and $G^{\prime \prime}\left[e^{\prime \prime}\right]$ participates on the right in Dumbbell $\left(G^{\prime}\left[e^{\prime}\right], G^{\prime \prime}\left[e^{\prime \prime}\right]\right)$. (Strictly speaking, there could be two such graphs, but let us consider only one of them. For concreteness, if $e^{\prime}=\left(v^{\prime}, w^{\prime}\right)$ and $e^{\prime \prime}=\left(v^{\prime \prime}, w^{\prime \prime}\right)$ where $I D\left(v^{\prime}\right)<I D\left(w^{\prime}\right)$ and $I D\left(v^{\prime \prime}\right)<I D\left(w^{\prime \prime}\right)$, then the graph Dumbbell $\left(G^{\prime}\left[e^{\prime}\right], G^{\prime \prime}\left[e^{\prime \prime}\right]\right)$ contains the bridge edges $\left(v^{\prime}, v^{\prime \prime}\right)$ and $\left(w^{\prime}, w^{\prime \prime}\right)$. Create a collection $\mathcal{I}$ of inputs for our problem consisting of all the dumbbell graphs

$$
\begin{aligned}
\mathcal{I}=\left\{\operatorname{Dumbbell}\left(G^{\prime}\left[e^{\prime}\right], G^{\prime \prime}\left[e^{\prime \prime}\right]\right) \mid\right. & G^{\prime}\left[e^{\prime}\right], G^{\prime \prime}\left[e^{\prime \prime}\right] \in \mathcal{G}^{o p e n}, \\
& \left.I D\left(G^{\prime}\left[e^{\prime}\right]\right) \cap I D\left(G^{\prime \prime}\left[e^{\prime \prime}\right]\right)=\emptyset\right\} .
\end{aligned}
$$

Partition the collection of inputs $\mathcal{I}$ into classes as follows: for every two graphs $G^{\prime}, G^{\prime \prime} \in \mathcal{G}$, define the class $\mathcal{C}\left(G^{\prime}, G^{\prime \prime}\right)=\left\{\right.$ Dumbbell $\left.\left(G^{\prime}\left[e^{\prime}\right], G^{\prime \prime}\left[e^{\prime \prime}\right]\right) \mid e^{\prime}, e^{\prime \prime} \in E\left(G_{0}\right)\right\}$, consisting of the $m^{2}$ dumbbell graphs constructed from $G^{\prime}$ and $G^{\prime \prime}$. Finally, create a uniform distribution $\Psi$ on $\mathcal{I}$.

First, we would like to prove that every deterministic algorithm $D$ that achieves LE on every graph in collection $\mathcal{I}$, has expected message complexity $\Omega(m)$ on $\Psi$. Yao's minimax principle (cf. Lemma 3.2) then implies that the randomized algorithm $R$ has expected message complexity $\Omega(m)$ on some graph $G^{*}$ of $\mathcal{I}$. Since every graph in the collection $\mathcal{I}$ has $2 m$ edges, this implies a lower bound of $\Omega(m)$ for $R$.

To prove this, we define an intermediate problem on the input collection $\mathcal{I}$, called bridge crossing (BC). An algorithm for this problem is required to send a message on one of the two bridge edges connecting the two open graphs (from either direction). More precisely, any algorithm solving BC is allowed to start simultaneously at all nodes, and succeeds if during its execution, a message has crossed one of the two connecting bridge edges. (Note that in our model, the nodes are unaware of their neighbors' identities, and in particular, the four nodes incident to the two bridge edges are unaware of this fact.)

Lemma 3.2 (Yao's Minimax Principle, cf. Prop. 2.6 in [18]). Consider a finite collection of inputs $\mathcal{I}$ and a distribution $\Psi$ on it. Let $X$ be the minimum expected cost of any deterministic algorithm that succeeds on at least a $1-2 \beta$ fraction of the graphs in the collection $\mathcal{I}$, for some positive constant $\beta$. Then $X / 2$ lower bounds the expected cost of any randomized algorithm $R$ on the worst-case graph of $\mathcal{I}$ that succeeds with probability at least $1-\beta$.

Basic counting yields the following:

Fact 3.3. Let the node degrees in $G_{0}$ be $d_{1}, \ldots, d_{n}$, where $d_{i}=\operatorname{deg}\left(v_{i}, G_{0}\right)$ for $1 \leqslant i \leqslant n$.

(a) The number of different possible port assignments is $K=\prod_{i=1}^{n} d_{i}$ !.

(b) The number of different possible ID assignments is $\left(\begin{array}{c}n^{4} \\ n\end{array}\right)$.

(c) The number of graphs in the collection $\mathcal{G}$ is $g=K \cdot\left(\begin{array}{c}n^{4} \\ n\end{array}\right)$.

(d) The number of graphs in the collection $\mathcal{G}^{\text {open }}$ is $g^{\text {open }}=g \cdot m$.

(e) The number of graphs in each class $\mathcal{C}\left(G^{\prime}, G^{\prime \prime}\right)$ is $m^{2}$.

(f) For every graph $G^{\prime} \in \mathcal{G}$, the number of graphs $G^{\prime \prime} \in \mathcal{G}$ ID disjoint from $G^{\prime}$ is $\tilde{g}=K \cdot\left(\begin{array}{c}n^{4}-n \\ n\end{array}\right)$.

(g) The number of classes $\mathcal{C}\left(G^{\prime}, G^{\prime \prime}\right)$ in the input collection $\mathcal{I}$ is $\tilde{h}=g \cdot \tilde{g}$.

(h) The number of dumbbell graphs in the input collection $\mathcal{I}$ is $\tilde{d}=\tilde{h} \cdot m^{2}$.

Also, straightforward calculations yield the following.

Lemma 3.4. $\tilde{g} \geqslant(1-1 / n) g$. 
Lemma 3.5. For every deterministic algorithm $A$ and for every two disjoint graphs $G^{\prime}, G^{\prime \prime} \in \mathcal{G}$, if $A$ achieves $B C$ on at least $\varepsilon m^{2}$ graphs in the class $\mathcal{C}\left(G^{\prime}, G^{\prime \prime}\right)$, for constant $0<\varepsilon \leqslant 1$, then the expected message complexity of $A$ on inputs taken from $\mathcal{C}\left(G^{\prime}, G^{\prime \prime}\right)$ with a uniform distribution is $\varepsilon^{2} m / 8=\Omega(m)$.

Proof. Consider two disjoint graphs $G^{\prime}, G^{\prime \prime} \in \mathcal{G}$ and an algorithm $A$ satisfying the premise of the lemma. Perform the following experiment. Run the code of algorithm $A$ on the nodes of the $2 n$-node graph $G^{\prime 2}$ composed of two (disconnected) copies of $G^{\prime}$. Denote this execution by $E X\left(G^{\prime}\right)$. (Of course, since $G^{\prime}$ is not a dumbbell graph, this is not a legal input for $A$, so there are no guarantees on the output or even termination of this execution.) The algorithm will send some messages, and then possibly halt. For each edge $e$ (interpreted as a directed edge), identify the first time $t(e)$ in which a message was sent over $e$ (in this direction) in this execution. Order the (directed) edges in increasing order of $t(e)$, getting the list $\hat{E}^{\prime}=\left(e_{1}^{\prime}, \ldots, e_{k^{\prime}}^{\prime}\right)$. (Edges on which no messages were sent are not included in this list, so $k^{\prime} \leqslant 2 m$.) Run a similar experiment $E X\left(G^{\prime \prime}\right)$ on $G^{\prime \prime}$, getting a list $\hat{E}^{\prime \prime}=\left(e_{1}^{\prime \prime}, \ldots, e_{k^{\prime \prime}}^{\prime \prime}\right)$.

Now consider the $m^{2}$ executions $E X\left(\operatorname{Dumbbell}\left(G^{\prime}\left[e^{\prime}\right], G^{\prime \prime}\left[e^{\prime \prime}\right]\right)\right)$ of $A$ on the dumbbell graphs in the class $\mathcal{C}\left(G^{\prime}, G^{\prime \prime}\right)$. In at least $\varepsilon m^{2}$ of these executions, the algorithm $A$ succeeds, so (at least) one message crosses one bridge in one direction. Without loss of generality, in at least $\varepsilon \mathrm{m}^{2} / 2$ of these executions, the first crossing message was sent from $G^{\prime}$ to $G^{\prime \prime}$.

Partition the dumbbell graphs in the class $\mathcal{C}\left(G^{\prime}, G^{\prime \prime}\right)$ into subclasses $C_{0}, C_{1}, \ldots, C_{k^{\prime}}$, where the subclass $C_{i}$ for $1 \leqslant i \leqslant k^{\prime}$ contains all the dumbbell graphs Dumbbell $\left(G^{\prime}\left[e^{\prime}\right], G^{\prime \prime}\left[e^{\prime \prime}\right]\right)$ in which $e^{\prime}=e_{i}^{\prime}$ and in execution $\operatorname{EX}\left(\operatorname{Dumbbell}\left(G^{\prime}\left[e^{\prime}\right], G^{\prime \prime}\left[e^{\prime \prime}\right]\right)\right)$, the first crossing message went over the edge $e_{i}^{\prime}$ from $G^{\prime}\left[e^{\prime}\right]$ to $G^{\prime \prime}\left[e^{\prime \prime}\right]$. The subclass $C_{0}$ contains all the remaining graphs of the class $\mathcal{C}\left(G^{\prime}, G^{\prime \prime}\right)$.

Consider an execution $\operatorname{EX}\left(\operatorname{Dumbbell}\left(G^{\prime}\left[e^{\prime}\right], G^{\prime \prime}\left[e^{\prime \prime}\right]\right)\right)$ in which BC was achieved, and assuming that the first crossing message was sent in round $t$ from $G^{\prime}$ to $G^{\prime \prime}$, say, over port $p$ that in the original $G^{\prime}$ was used for $e^{\prime}$. A crucial observation is that the part of this execution restricted to $G^{\prime}\left[e^{\prime}\right]$ is identical to the execution $E X\left(G^{\prime}\right)$ up to and including round $t$. Similarly, the part of this execution restricted to $G^{\prime \prime}\left[e^{\prime \prime}\right]$ is identical to the execution $E X\left(G^{\prime \prime}\right)$ up to and including round $t$. This implies that $e^{\prime}$ must occur in the list $\hat{E}^{\prime}$ in some position, as $e_{j}^{\prime}$. In particular, it follows that the subclass $C_{0}$ contains only dumbbell graphs in which the first crossing message went from $G^{\prime \prime}\left[e^{\prime \prime}\right]$ to $G^{\prime}\left[e^{\prime}\right]$ plus all the dumbbell graph in which no message crossed. In addition, it follows that $\left|C_{0}\right| \leqslant(1-\varepsilon) m^{2} / 2$ and $\sum_{i=1}^{k^{\prime}}\left|C_{i}\right| \geqslant \varepsilon m^{2} / 2$. Moreover, in the execution $\operatorname{EX}\left(\operatorname{Dumbbell}\left(G^{\prime}\left[e^{\prime}\right], G^{\prime \prime}\left[e^{\prime \prime}\right]\right)\right)$, algorithm $A$ must have sent at least one message on each of the edges $e_{i}^{\prime}$ for $1 \leqslant i \leqslant j$, i.e., $A$ must have sent at least $j$ messages.

We define $\ell_{j}=\left|C_{j}\right|$ to be the number of executions $\operatorname{EX}\left(\operatorname{Dumbbell}\left(G^{\prime}\left[e^{\prime}\right], G^{\prime \prime}\left[e^{\prime \prime}\right]\right)\right)$ in which the first crossing message was sent from $G^{\prime}$ to $G^{\prime \prime}$ over the edge $e_{j}^{\prime}$. This requires, in particular, that $e^{\prime}=e_{j}^{\prime}$. As there are exactly $m$ dumbbell graphs $\operatorname{Dumbbell}\left(G^{\prime}\left[e_{j}^{\prime}\right], G^{\prime \prime}\left[e^{\prime \prime}\right]\right)$, it follows that $\ell_{j} \leqslant m$. Let $B=\sum_{j=1}^{k^{\prime}} \ell_{j}$. By assumption, $B \geqslant \varepsilon m^{2} / 2$. Let $Q$ be the total number of messages sent by $A$ in all these executions. Then $Q \geqslant \sum_{j=1}^{k^{\prime}} \ell_{j} \cdot j$. To lower bound $Q$, note that this last sum is minimized if the first $B / m$ summands are $\ell_{i}=m$, for $i=1, \ldots, B / m$, and the remaining summands are 0 . Hence

$$
Q \geqslant \sum_{j=1}^{B / m} m \cdot j \geqslant \frac{m}{2} \cdot \frac{B}{m} \cdot\left(\frac{B}{m}+1\right) \geqslant B^{2} /(2 m) \geqslant \varepsilon^{2} m^{3} / 8 .
$$

Therefore the expected cost incurred by $A$ over the class $\mathcal{C}\left(G^{\prime}, G^{\prime \prime}\right)$ is at least $\varepsilon^{2} m / 8=\Omega(m)$. 
Lemma 3.6. Every deterministic algorithm $A$ that achieves $B C$ on at least $1 / 4$ of the dumbbell graphs in the collection $\mathcal{I}$ has expected message complexity $\Omega(m)$ on $\Psi$.

Proof. Consider an algorithm $A$ as in the lemma. Let $z$ denote the number of dumbbell graphs in the collection $\mathcal{I}$ on which algorithm $A$ achieves BC. By the assumption of the lemma, $z \geqslant \tilde{d} / 4$. Let $X$ denote the set of pairs of disjoint graphs $\left(G^{\prime}, G^{\prime \prime}\right)$ such that algorithm $A$ achieves BC on at least $m^{2} / 8$ of the $m^{2}$ dumbbell graphs Dumbbell $\left(G^{\prime}\left[e^{\prime}\right], G^{\prime \prime}\left[e^{\prime \prime}\right]\right)$ in the class $\mathcal{C}\left(G^{\prime}, G^{\prime \prime}\right)$. Let $Y$ denote the set of remaining pairs (such that $A$ achieves BC on fewer than $m^{2} / 8$ of the dumbbell graphs in $\left.\mathcal{C}\left(G^{\prime}, G^{\prime \prime}\right)\right)$. Let $x=|X|$ and $y=|Y|$. Note that $x+y=\tilde{h}$.

Claim 3.7. $x \geqslant \tilde{h} / 8$.

Proof. Observe that $z$, the number of dumbbell graphs in $\mathcal{I}$ on which algorithm $A$ achieves BC, cannot exceed $x m^{2}+y m^{2} / 8$, hence

$$
x m^{2}+y m^{2} / 8 \geqslant z \geqslant \tilde{d} / 4=\tilde{h} m^{2} / 4 .
$$

Hence assuming, to the contrary, that $x<\tilde{h} / 8$, implies that

$$
\frac{\tilde{h}}{8} \cdot m^{2}+\frac{7 \tilde{h}}{8} \cdot \frac{m^{2}}{8}>x m^{2}+y m^{2} / 8 \geqslant \frac{\tilde{h} m^{2}}{4}
$$

or $15 / 64>1 / 4$, contradiction.

By Lemma 3.5, for every pair of disjoint graphs $\left(G^{\prime}, G^{\prime \prime}\right) \in X$, the expected message complexity of $A$ on inputs taken from $\mathcal{C}\left(G^{\prime}, G^{\prime \prime}\right)$ with a uniform distribution is at least $m / 2^{7}$. Hence the total number of messages sent by the algorithm when executed over all inputs from $\mathcal{C}\left(G^{\prime}, G^{\prime \prime}\right)$ is at least, $\left(x m^{2}\right) \cdot m / 2^{7}+\left(y m^{2}\right) \cdot 0$. The first summand stands for the $x m^{2}$ graphs in the $x$ classes of $X$, and the second summand stands for the graphs in the classes of $Y$. By Claim 3.7, this is at least $\left(\tilde{h} / 2^{3}\right) \cdot\left(m^{3} / 2^{7}\right)=\tilde{d} m / 2^{10}$, hence the expected message complexity of algorithm $A$ over all disjoint graph pairs in $\mathcal{I}$ (with a uniform distribution) is at least $m / 2^{10}=\Omega(m)$.

Lemma 3.8. Let $\varepsilon$ and $\delta \geqslant 1 / 4$ be positive constants such that $7 \varepsilon+\delta \leqslant 1$. If a deterministic universal $L E$ algorithm $A$ solves $L E$ on at least a $1-\varepsilon$ fraction of the input graphs in $\mathcal{I}$, then $A$ achieves $B C$ on at least a $\delta$ fraction of the graphs in $\mathcal{I}$.

Proof. Denote by $\mathcal{I}^{L E}$ (respectively, $\mathcal{I}^{B C}$ ) the set of input dumbbell graphs on which algorithm $A$ achieves LE (resp., BC). Let $\mathcal{I}^{*}=\mathcal{I}^{L E} \backslash \mathcal{I}^{B C}$. By the assumption of the lemma, $\left|\mathcal{I}^{L E}\right| \geqslant(1-\varepsilon) \tilde{d}$. Assume, towards contradiction, that $\left|\mathcal{I}^{B C}\right|<\delta \tilde{d}$. Then

$$
\left|\mathcal{I}^{*}\right|>(1-\varepsilon-\delta) \tilde{d}
$$

Let $W$ denote the set of open graphs $G^{\prime}\left[e^{\prime}\right]$ that participate on the left in dumbbell graphs in $\mathcal{I}^{*}$. Formally,

$$
W=\left\{G^{\prime}\left[e^{\prime}\right] \in \mathcal{G}^{\text {open }} \mid \exists G^{\prime \prime}\left[e^{\prime \prime}\right] \text { s.t. }\left(G^{\prime}\left[e^{\prime}\right], G^{\prime \prime}\left[e^{\prime \prime}\right]\right) \in \mathcal{I}^{*}\right\} .
$$

Let $Z=\mathcal{G} \backslash W$. Note that $|W|+|Z|=g m$. Observe that

$$
(1-\varepsilon-\delta) g \tilde{g} m^{2}=(1-\varepsilon-\delta) \tilde{d}<\left|\mathcal{I}^{*}\right| \leqslant|W| \tilde{g} m .
$$

The last inequality follows from the fact that we can combine $G^{\prime}\left[e^{\prime}\right] \in W$ to form a dumbbell with any $G^{\prime \prime}\left[e^{\prime \prime}\right]$ of the $\tilde{g}$ disjoint graphs where $e^{\prime \prime}$ can be any one of $m$ edges. 
Observation 3.9. $|W|>(1-\varepsilon-\delta) g m$.

Corollary 3.10. $|Z|<(\varepsilon+\delta) g m$.

Consider an execution of algorithm $A$ on a dumbbell graph $\left(G^{\prime}\left[e^{\prime}\right], G^{\prime \prime}\left[e^{\prime \prime}\right]\right) \in \mathcal{I}^{*}$. Necessarily, in one of the two graphs, all nodes ended in state NON-ELECTED, and in the other graph, exactly one node ended in state ELECTED and all the others in state NON-ELECTED. Suppose all nodes in $G^{\prime}\left[e^{\prime}\right]$ ended in state NON-ELECTED. Then for every other dumbbell graph $\left(G^{\prime}\left[e^{\prime}\right], G^{\prime \prime \prime}\left[e^{\prime \prime \prime}\right]\right) \in \mathcal{I}^{*}$ or $\left(G^{\prime \prime \prime}\left[e^{\prime \prime \prime}\right], G^{\prime}\left[e^{\prime}\right]\right) \in \mathcal{I}^{*}$ in which $G^{\prime}\left[e^{\prime}\right]$ participates, the run on $G^{\prime}\left[e^{\prime}\right]$ will behave the same as in the run on $\left(G^{\prime}\left[e^{\prime}\right], G^{\prime \prime}\left[e^{\prime \prime}\right]\right)$, so all nodes in $G^{\prime}\left[e^{\prime}\right]$ will end in state NON-ELECTED.

This observation implies that the open graphs in $W$ can be partitioned into two sets:

- the set $W_{N E}$ of graphs $G^{\prime}\left[e^{\prime}\right] \in W$ for which in executions on dumbbell graphs belonging to $\mathcal{I}^{*}$, all nodes in $G^{\prime}\left[e^{\prime}\right]$ end in state NON-ELECTED, and

- the set $W_{E}$ of graphs $G^{\prime}\left[e^{\prime}\right] \in W$ for which in executions on dumbbell graphs belonging to $\mathcal{I}^{*}$, exactly one node in $G^{\prime}\left[e^{\prime}\right]$ ends in state ELECTED and all other nodes end in state NON-ELECTED.

For every open graph $G^{\prime}\left[e^{\prime}\right] \in W_{N E}$, let

$$
\Gamma\left(G^{\prime}\left[e^{\prime}\right]\right)=\left\{G^{\prime \prime}\left[e^{\prime \prime}\right] \in \mathcal{G}^{\text {open }} \mid G^{\prime}\left[e^{\prime}\right] \text { and } G^{\prime \prime}\left[e^{\prime \prime}\right] \text { are ID disjoint }\right\} .
$$

Also let

$$
\begin{aligned}
\Gamma_{N E}\left(G^{\prime}\left[e^{\prime}\right]\right) & =W_{N E} \cap \Gamma\left(G^{\prime}\left[e^{\prime}\right]\right), \\
\Gamma_{E}\left(G^{\prime}\left[e^{\prime}\right]\right) & =W_{E} \cap \Gamma\left(G^{\prime}\left[e^{\prime}\right]\right), \\
\Gamma_{Z}\left(G^{\prime}\left[e^{\prime}\right]\right) & =Z \cap \Gamma\left(G^{\prime}\left[e^{\prime}\right]\right) .
\end{aligned}
$$

Denote the sizes of these sets by $\gamma\left(G^{\prime}\left[e^{\prime}\right]\right), \gamma_{N E}\left(G^{\prime}\left[e^{\prime}\right]\right), \gamma_{E}\left(G^{\prime}\left[e^{\prime}\right]\right)$, and $\gamma_{Z}\left(G^{\prime}\left[e^{\prime}\right]\right)$, respectively. Note that

$$
\gamma_{N E}\left(G^{\prime}\left[e^{\prime}\right]\right)+\gamma_{E}\left(G^{\prime}\left[e^{\prime}\right]\right)+\gamma_{Z}\left(G^{\prime}\left[e^{\prime}\right]\right)=\gamma\left(G^{\prime}\left[e^{\prime}\right]\right)=\tilde{g} m .
$$

We separate the analysis into two cases.

Case 1: $\left|W_{N E}\right|>|W| / 2$ : By Obs. 3.9, $\left|W_{N E}\right|>(1-\varepsilon-\delta) g m / 2$. By the assumption of Case 1, $\gamma_{E}\left(G^{\prime}\left[e^{\prime}\right]\right) \leqslant\left|W_{E}\right| \leqslant|W| / 2 \leqslant g m / 2$. By Cor. 3.10, $\gamma_{Z}\left(G^{\prime}\left[e^{\prime}\right]\right) \leqslant|Z|<(\varepsilon+\delta) g m$. Combining the last two facts with Eq. (3) and Lemma 3.4, implies that

$$
\begin{aligned}
\gamma_{N E}\left(G^{\prime}\left[e^{\prime}\right]\right) & =\tilde{g} m-\left(\gamma_{E}\left(G^{\prime}\left[e^{\prime}\right]\right)+\gamma_{Z}\left(G^{\prime}\left[e^{\prime}\right]\right)\right) \\
& \geqslant(1-1 / n) g m-g m / 2=(1 / 2-1 / n) g m
\end{aligned}
$$

Our key observation is that every pair of open graphs from $W_{N E}$ forms a dumbbell graph on which algorithm $A$ fails to solve LE, since no node will end in state ELECTED. This allows us to lower bound the number $X$ of dumbbell graphs on which agorithm $A$ fails to solve leader election: summing over all graphs in $W_{N E}$, we get that

$$
\begin{aligned}
X & \geqslant \sum_{G^{\prime}\left[e^{\prime}\right] \in W_{N E}} \gamma_{N E}\left(G^{\prime}\left[e^{\prime}\right]\right) \geqslant\left|W_{N E}\right| \cdot(1 / 2-1 / n) g m \\
& >((1-\varepsilon-\delta) g m / 2) \cdot((1 / 2-1 / n) g m) \\
& \geqslant(1-\varepsilon-\delta) \tilde{d} / 6 .
\end{aligned}
$$


On the other hand, recall that we have assumed that $\left|\mathcal{I}^{L E}\right| \geqslant(1-\varepsilon) \tilde{d}$, so $X \leqslant \varepsilon \tilde{d}$. It follows that $(1-\varepsilon-\delta) \tilde{d} / 6<\varepsilon \tilde{d}$. Rearranging, we get that $7 \varepsilon+\delta>1$, contradicting the assumption of the lemma.

Case 2: $\left|W_{E}\right|>|W| / 2$ : A similar contradiction is derived, based on the observation that every pair of open graphs from $W_{E}$ forms a dumbbell graph on which algorithm $A$ fails to solve LE.

This completes the proof of Lemma 3.8.

Combining Lemmas 3.6 and 3.8 allows us to use a reduction of BC to LE to show the claimed result for a universal randomized algorithm $R$ that achieves LE with probability at least $1-\beta$ : Suppose that $A$ is a universal deterministic leader election algorithm that achieves LE on at least a $1-2 \beta$ fraction of the dumbbell graphs in $\mathcal{I}$. By Lemma 3.8, we know that $A$ achieves $\mathrm{BC}$ on at least a $\delta \geqslant 1 / 4$ fraction of the dumbbell graphs in $\mathcal{I}$. Applying Lemma 3.6 yields that $A$ must have an expected message complexity of $\Omega(m)$ on distribution $\Psi$, which shows the theorem for deterministic algorithms. By a simple application of Yao's minimax principle (cf. Lemma 3.2), it follows that the $\Omega(m)$ bound for deterministic algorithms is a lower bound for the expected message complexity of $R$ (under the worst case input), thereby completing the proof of Theorem 3.1.

At this point, let us explain why the above proof fails for weaker algorithms, which are guaranteed to work correctly only when the nodes know $D$. The problem occurs in the proof of Lemma 3.5. In that proof, we run an experiment where we execute algorithm $A$ (which is now assumed to work correctly only when the nodes are given the diameter $\operatorname{Diam}(\mathrm{G})$ as part of their inputs) on an "illegal" graph $G^{\prime 2}$ composed of two (disconnected) copies of $G^{\prime}$. We then argue that the execution on the dumbbell graphs serving as the "real" inputs will behave the same as on the illegal graph $G^{2}$ (so long as there was no bridge crossing). But this claim no longer holds when the nodes get the diameter $D$ as part of their input, since the diameter of the dumbbell graph is different from that of the illegal graph $G^{\prime 2}$ (which is infinite), so a node $v$ will see a different input in the two execution.

To fix this problem, a natural idea would be to "feed" the nodes participating in the experiment on the illegal graph $G^{2}$ a "fake" input on the diameter, i.e., set the input variable $D I A M_{v}$ at each node $v$ to $D^{\prime}$, where $D^{\prime}$ is the diameter of the dumbbell graph. (Again, as this input is illegal, it is not guaranteed that the algorithm will generate a meaningful output, but still, the execution will behave the same as in the "real" execution on the dumbbell graph.)

A technical difficulty that prevents us from using this idea directly is that there are many dumbbell graphs for a given pair $G^{\prime}, G^{\prime \prime}$, and they have different diameters. This means that no single experiment (run with a specific value of $D$ fed to the nodes) will be similar to all executions on all dumbbell graphs.

Hence to overcome this difficulty, it is necessary to pick $G_{0}$ and construct the collection of input graphs for the algorithm so that no matter which graphs $G^{\prime}$ and $G^{\prime \prime}$ are chosen, and which edges $e^{\prime}$ and $e^{\prime \prime}$ are crossed-over, the diameter of the resulting dumbbell graph is always the same. The observation that assists us in achieving this property is that we are free to select the graph $G_{0}$, so long as we adhere to the requirements that its size is $n$ nodes and $\Theta(m)$ edges. So let us pick $G_{0}$ to have the following structure. Let $\kappa$ be the largest integer such that $\left(\begin{array}{c}\kappa \\ 2\end{array}\right)+\kappa \leqslant m$. Let $G_{0}^{1}$ be the complete graph on $\kappa$ nodes, with $m_{1}=\left(\begin{array}{c}\kappa \\ 2\end{array}\right)$ edges. Let $G_{0}^{2}$ be a path of $n-\kappa$ nodes, $\left(b_{1}, \ldots, b_{n-\kappa}\right)$. Combine the two graphs into $G_{0}$ by adding $\kappa$ edges connecting $b_{1}$ to every node in $G_{0}^{1}$. It is straightforward to verify that the resulting graph $G_{0}$ satisfies the size requirements.

Next, we modify the proof by limiting the ways in which we create open graphs from $G_{0}$. Specifically, we will consider only open graphs obtained by disconnecting an edge $e^{\prime}$ in the clique 
$G_{0}^{1}$. That is, the resulting family of open graphs will contain only graphs $\left(G^{\prime}\left[e^{\prime}\right]\right)$ for $e^{\prime} \in E\left(G_{0}^{1}\right)$.

The key observation is that no matter which two edges $e^{\prime}$ and $e^{\prime \prime}$ of $G_{0}^{1}$ we choose to disconnect in $G^{\prime}$ and $G^{\prime \prime}$ respectively, the resulting dumbbell graph Dumbbell $\left(G^{\prime}\left[e^{\prime}\right], G^{\prime \prime}\left[e^{\prime \prime}\right]\right)$ will always have the same diameter, $D=2 n-2 \kappa+1$ (which is the distance between the two endpoints $b_{n-\kappa}$ of the two graphs $G^{\prime}\left[e^{\prime}\right]$ and $\left.G^{\prime \prime}\left[e^{\prime \prime}\right]\right)$.

One can verify that with this change, the rest of the proof goes through, with $m_{1}$ replacing $m$ in the intermediate claims. In particular, the number of graphs in each class $\mathcal{C}\left(G^{\prime}, G^{\prime \prime}\right)$ becomes $m_{1}^{2}$, and so on. We end up proving that any deterministic leader election algorithm uses an average of $\Omega\left(m_{1}\right)$ messages on the collection $\mathcal{I}$ of inputs. Those messages are sent over the edges of the $\kappa$-clique (we cannot prove that there will be any messages sent over the edges of the paths $G_{0}^{2}$ in either side of the dumbbell graphs). Yet as $m_{1}=\Omega(m)$, this suffices to establish the desired lower bound of $\Omega(m)$ on the expected message complexity of $R$ on the worst case input.

\subsection{A Lower Bound on the Message Complexity of Broadcast}

We can leverage our lower bound technique to show an analogous bound for the broadcast problem [5], where a single node must convey a message to all other nodes. In fact, we can show a lower bound of $\Omega(m)$ messages even for the weaker majority broadcast problem, where the message of a single node needs to reach $>n / 2$ nodes.

Consider the same collection of dumbbell graphs $\mathcal{I}$ as in Lemma 3.6. If a deterministic algorithm $B$ successfully broadcasts in some execution on a graph $G \in \mathcal{I}$, then $B$ also achieves bridge crossing in $G$. The following is immediate from Lemma 3.6:

Lemma 3.11. Suppose that there is an algorithm $B$ that achieves broadcast on at least $1 / 4$ of the graphs in $\mathcal{I}$. Then $B$ has expected message complexity of $\Omega(m)$ on $\Psi$.

By a direct application of Lemma 3.2, we get a lower bound for randomized algorithms:

Corollary 3.12. Let $R^{\prime}$ be an algorithm that successfully broadcasts a message from a source node to a majority of nodes with probability at least $1-\beta$, for some constant $\beta \leqslant 3 / 8$. For every sufficiently large $n$ and $n \leqslant m \leqslant\left(\begin{array}{l}n \\ 2\end{array}\right)$, there exists a (connected) graph $G$ of $n$ nodes and $\Theta(m)$ edges, such that the expected number of messages used by $R^{\prime}$ on $G$ is $\Omega(m)$. The above holds even if $n, m$ and $D$ are known to the algorithm and all nodes wake up simultaneously.

\subsection{Time Complexity Lower Bound}

Theorem 3.13. Consider a universal leader election algorithm $R$ that succeeds with probability $1-\beta$, assuming that $\beta<1 / 16$ in the anonymous setting and $\beta<1 / 16-15 / 16 n^{2}$, if nodes have unique ids. Then, for every $n$ and every nondecreasing function $D(n)$ with $2<D(n)<n$, there exists a graph $G$ of $n^{\prime} \in \Theta(n)$ nodes and diameter $D^{\prime} \in \Theta(D(n))$ where $R$ takes $\Omega\left(D^{\prime}\right)$ rounds with constant probability. This is true even if all nodes know $n^{\prime}$ and $D^{\prime}$, and wake up simultaneously.

Proof. For a given $n$ and $D(n)$, we construct the following lower bound graph $G$ : Let $D^{\prime}=$ $4\lceil D(n) / 4\rceil$ and let $n^{\prime}=\gamma D^{\prime}$, where $\gamma>0$ is the smallest integer such that $\gamma D^{\prime} \geqslant n$; clearly $n^{\prime} \in \Theta(n)$. The graph $G$ contains $D^{\prime}$ cliques, each of size $\gamma$. Partition the cliques into 4 subgraphs $C_{0}, \ldots, C_{3}$ referred to as arcs, each containing $D^{\prime} / 4$ cliques. Let $c_{i, j}$ denote the $j$-th clique in the $i$-th arc and let $v_{i, j, k}$ be the $k$-th node in $c_{i, j}$, for $0 \leqslant i \leqslant 3,0 \leqslant j \leqslant D^{\prime} / 4-1$, and $0 \leqslant k \leqslant \gamma-1$. The 


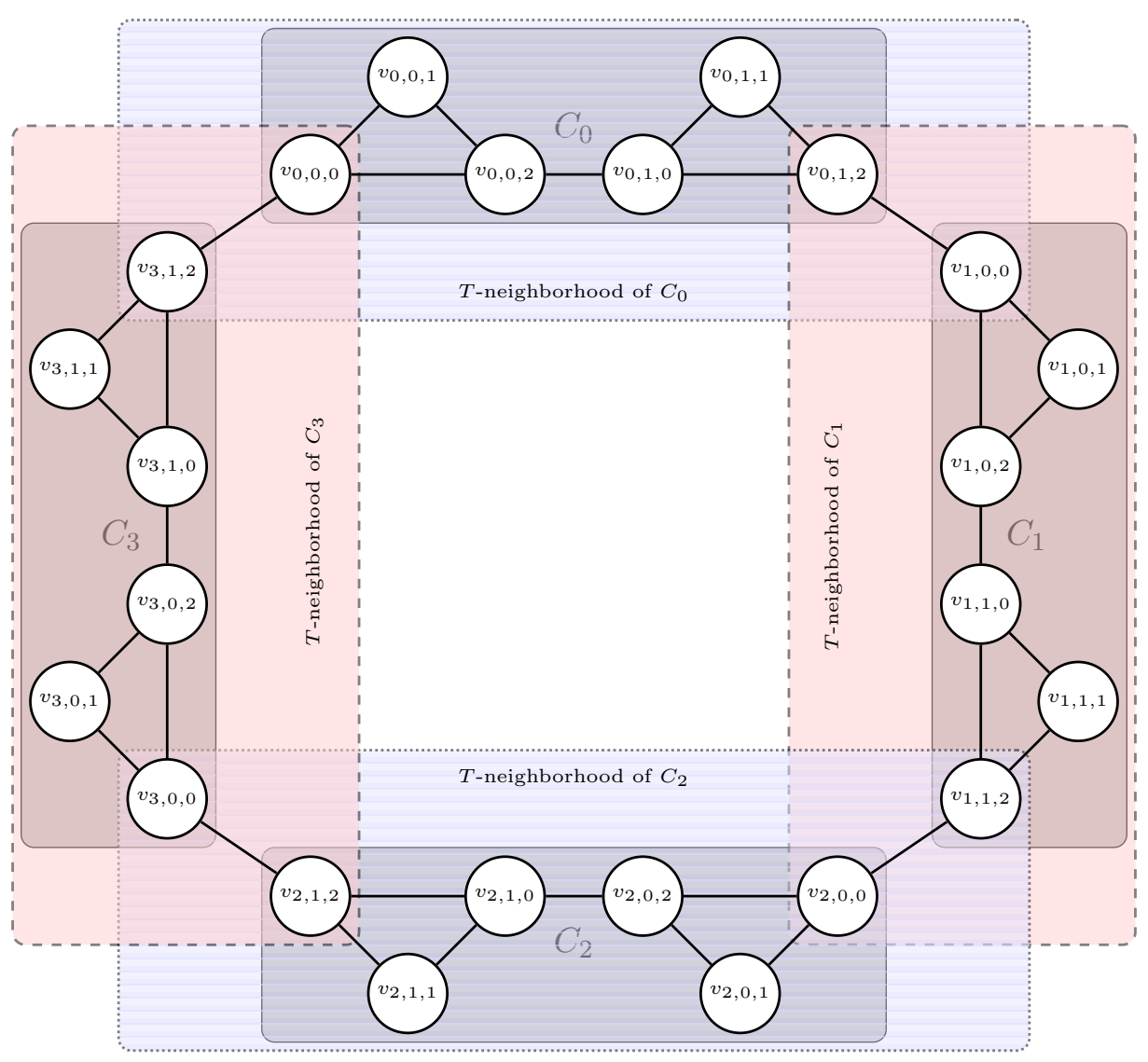

Figure 1: The Clique-Cycle Construction of Theorem 3.13 for $D^{\prime}=8$ and $n^{\prime}=24$. The blue dotted rectangles form $H_{Z}$ and the red dashed rectangles correspond to $H_{Z^{\prime}}$.

graph $G$ is a cycle $C$ formed by the $D^{\prime}$ cliques connected the following way: For every $i$ (adhering to the range defined above) and every $j \leqslant D^{\prime} / 4-2$, the edge $\left(v_{i, j, \gamma-1}, v_{i, j+1,0}\right)$ connects the clique $c_{i, j}$ to the clique $c_{i, j+1}$ within the same $\operatorname{arc} C_{i}$. The connections between $\operatorname{arcs} C_{i}$ and $C_{i+1} \bmod 4$ are given by the edges $\left.\left(v_{i, D^{\prime} / 4-1, \gamma-1}, v_{(i+1} \bmod 4\right), 0,0\right)$. See Figure 1 for an example.

We first consider the anonymous case, where each node starts in the same initial state. Let $T$ be the random variable denoting the running time of the assumed algorithm $R$ and assume that the event $T \in o\left(D^{\prime}\right)$ happens with probability $\delta=1-o(1)$. In the remainder of the proof we will show that this yields a contradiction.

Consider the two sets of nodes $Z=C_{0} \cup C_{2}$ and $Z^{\prime}=C_{1} \cup C_{3}$ formed by non-adjacent arcs. Let $\phi: V(G) \rightarrow V(G)$ be a mapping such that $\left.\phi\left(v_{i, j, k}\right)=v_{(i+1} \bmod 4\right), j, k$. Let $H_{Z}$ be the subgraph consisting of $Z$ and its $T$-neighborhood and define $H_{Z^{\prime}}$ analogously. By the definition of the adjacencies of $C$, it follows that the subgraph induced by $\phi\left(V\left(H_{Z}\right)\right)$ is isomorphic to $H_{Z^{\prime}}$.

Let configuration $\mathcal{C}=\left\langle\sigma_{1}, \ldots, \sigma_{\ell}\right\rangle$ denote a vector where each entry $\sigma_{i}$ denotes a potential local state of a node. For a set of nodes $S=\left\{u_{1}, \ldots, u_{\ell}\right\}$, we say that $S$ realizes $\mathcal{C}$ in round $r$, if node $u_{i}$ is in state $\sigma_{i}$ in round $r$; let $E(\mathcal{C}, S, r)$ denote the event that this happens.

Claim 3.14. Let $S$ be any set of nodes. For any round $r$ and any configuration $\mathcal{C}$, we have 
$\mathbb{P}\left[E(\mathcal{C}, S, r) \mid T \in o\left(D^{\prime}\right)\right]=\mathbb{P}\left[E(\mathcal{C}, \phi(S), r) \mid T \in o\left(D^{\prime}\right)\right]$

Proof. Recalling that initially each node is in the exact same state, it follows that, for any configuration $\mathcal{C}$, and any set of nodes $S$, we have $\mathbb{P}[E(\mathcal{C}, S, 1)]=\mathbb{P}[E(\mathcal{C}, \phi(S), 1)]$. This is true because node $v_{i, j, k} \in S$ observes the same neighborhood as node $v_{i+1} \bmod 4, j, k$ and each neighbor $v$ of $v_{i, j, k}$ is in the same state as neighbor $\phi(v)$ of $v_{i+1} \bmod 4, j, k$. Thus, by inductively applying this symmetry argument, the mapping $\phi$ induces equi-probable realizations of any local configuration $\mathcal{C}$, in any round $r$.

Note that we do not claim independence of the events $E(\mathcal{C}, Z, r)$ and $E\left(\mathcal{C}, Z^{\prime}, r\right)$. Let $L$ (resp., $L^{\prime}$ ) be the event that one node in $Z$ (resp., $Z^{\prime}$ ) becomes leader and let $L_{i}$ be the event that there is one leader elected in arc $C_{i}$. By Claim 3.14, it holds that $\mathbb{P}\left[L \mid T \in o\left(D^{\prime}\right)\right]=\mathbb{P}\left[L^{\prime} \mid T \in o\left(D^{\prime}\right)\right]$; let $q$ denote this probability. If the algorithm terminates in $T=o(D)=o\left(D^{\prime}\right)$ rounds, then there is no causal influence between $C_{0}$ and $C_{2}$, which means that $E\left(\mathcal{C}, C_{0}, r\right)$ and $E\left(\mathcal{C}, C_{2}, r\right)$ are stochastically independent (as opposed to events $E(\mathcal{C}, Z, r)$ and $E\left(\mathcal{C}, Z^{\prime}, r\right)$ above!), for any configuration $\mathcal{C}$ and any round $r \leqslant T$. In particular, this includes all configurations that satisfy $L_{i}$. Let $p=\mathbb{P}\left[L_{0} \mid T \in o\left(D^{\prime}\right)\right]$; again, from Claim 3.14, we know that $\mathbb{P}\left[L_{0} \mid T \in o\left(D^{\prime}\right)\right]=\cdots=$ $\mathbb{P}\left[L_{3} \mid T \in o\left(D^{\prime}\right)\right]$, and due to independence of $L_{0}$ and $L_{2}$, we also have $q=2 p(1-p)$. Let OneLd be the event that the algorithm elects 1 leader. We know that

$$
\begin{aligned}
\mathbb{P}\left[\text { OneLd } \mid T \in o\left(D^{\prime}\right)\right] & =\frac{\mathbb{P}[\text { OneLd }]}{\mathbb{P}\left[T \in o\left(D^{\prime}\right)\right]}-\frac{\mathbb{P}\left[\text { OneLd } \mid T \in \Omega\left(D^{\prime}\right)\right] \mathbb{P}\left[T \in \Omega\left(D^{\prime}\right)\right]}{\mathbb{P}\left[T \in o\left(D^{\prime}\right)\right]} \\
& \geqslant \frac{1-\beta-(1-\delta)}{\delta}=1-\frac{\beta}{\delta},
\end{aligned}
$$

and clearly

$$
\mathbb{P}\left[\text { OneLd } \mid T \in o\left(D^{\prime}\right)\right] \leqslant \mathbb{P}\left[L \mid T \in o\left(D^{\prime}\right)\right]+\mathbb{P}\left[L^{\prime} \mid T \in o\left(D^{\prime}\right)\right]=2 q .
$$

Thus $q \geqslant \frac{1}{2}(1-\beta / \delta)$, which means that $p \geqslant \frac{1}{2}(1-\sqrt{\beta / \delta})$. We know that $\beta=\mathbb{P}[$ error $] \geqslant p^{2}$ and thus $\frac{1}{4}(1-\sqrt{\beta / \delta})^{2} \leqslant \beta$. Note that since $\beta<1 / 16$, this inequality requires $\delta<1 / 4$ and therefore $T \in \Omega\left(D^{\prime}\right)$ with constant probability. This completes the proof for the anonymous case.

Finally, for the non-anonymous case we show a reduction to the anonymous case. Now suppose that $R$ is an algorithm designed for a setting where each node has a unique id and again assume that $R$ takes only $o(D)$ rounds with probability $1-o(1)$. Consider algorithm $R^{\prime}$ that extends algorithm $R$ by causing each node to choose an id uniformly at random from $\left[1, n^{4}\right]$ initially. This id is then used as input for algorithm $R$ (instead of the id assigned in a non-anonymous network in which $R$ is guaranteed to work). The event $U$, where all chosen ids are unique, occurs with probability at least $1-n^{-2}$. By definition of $R^{\prime}$, we have $\mathbb{P}\left[R^{\prime}\right.$ succeeds $\left.\mid U\right]=\mathbb{P}[R$ succeeds $]$ and, from the anonymous case above, we know that $\mathbb{P}\left[R^{\prime}\right.$ succeeds $] \leqslant 15 / 16$. It follows that $\mathbb{P}\left[R^{\prime}\right.$ succeeds $\left.\mid U\right] \leqslant(15 / 16) / \mathbb{P}[U] \leqslant 15 / 16\left(1-n^{-2}\right)$, for sufficiently large $n$. This shows that algorithm $R$ succeeds with probability at most $15 / 16+15 / 16 n^{2}$ and completes the proof of the Theorem.

\section{Algorithms}

In this section we present the technical details of the upper bounds of Table 1. (See Section 1.1 for a general overview.) 


\subsection{Demonstrating that the message lower bound is tight}

We first show that our $\Omega(m)$ lower bound (cf. Theorem 3.1) is tight even in the deterministic case by presenting a deterministic $O(m)$ messages algorithm. What allows this algorithm to use less messages than other algorithms in this paper is the fact that we do not bound the time. Fredrickson and Lynch [8] presented such an algorithm for rings (using $n$ messages; recall that in a ring, $m=n$ ). The algorithm presented below is a generalization of their algorithm.

For simplicity, we first assume all the nodes wake up at the same time. Each node $v$ initiates an annexing agent that walks over all the graph in a Depth First Search (DFS) manner, carrying $v$ 's unique ID. (Using agents is just a convenient way to describe such an algorithm [12]; the actual implementation is by messages; to translate to message passing, when the agent of $v$ wishes to pass over some edge $e$, a message, carrying $v$ 's ID, is sent over $e$ instead; the DFS marking of edge $e$ are left at the endpoints of $e$, and are associated there with the ports of $e$ ).

To take care of congestion, if two agents are waiting to traverse edge $e$, then only the agent with the lower ID traverses it. The one with the higher ID, is destroyed. Moreover, the ID of each agent who has ever passed any node $w$ is left in $w$. An agent for some ID $j$ who reaches a node previously visited by an agent with a smaller ID $i<j$ is destroyed. Similarly, if the agent for $j$ is waiting in some node $w$ when the agent for some $i<j$ arrives, then the agent for $j$ is destroyed.

The rule enabling the reduction in messages is that an agent whose ID is $i$ performs one DFS step each $2^{i}$ steps. An agent for ID $i$ who completed the DFS is back in the node whose unique ID is $i$. It declares this node the leader.

The message complexity is translated immediately to the total number of DFS steps taken by all the agents. The agent with the lowest ID takes $2 m$ steps ( $2 m$ messages), and covers the whole network. By that time, all the other agents have encountered the lowest id agent and have been destroyed. Out of those, the agent with the next to smallest ID took at most half of the number of steps taken by the lowest. That is, the next to lowest took at most $m$ steps. The next took, at most, $\frac{m}{2}$. The total number of steps taken by everybody is no larger than $4 m$.

The time complexity depends on the lowest ID $i$ (it is $2 m 2^{i}$ ). Each node may need to use memory (of $O(\log n)$ bits) per each other node and per each of its own ports (for multiple DFSs performed in parallel).

Let us now remove the assumption that all the nodes wake up simultaneously. For that, the algorithm starts with a "wakeup" phase as follows. Each node who wakes up spontaneously, starts a (synchronous) distributed Breadth First Search algorithm. Each not- yet- awake node who receives a message of the BFS, wakes up and forwards the BFS to its neighbors. An already awake node who receives such a message discards it. Clearly, all the nodes wake up, and the total cost of this phase is $2 m$ messages and $D$ time.

Having woken up, each node initiates an annexing agent who acts as described above for the annexing token. Hence, a leader is elected using no more than $4 m$ messages, after the time $t_{1}$ when all the nodes are awake. Let us analyze the number of messages used by annexing agents from the time $t_{0}$ when the first node (or set of nodes) woke up, until $t_{1}$. Recall that the duration of this time interval is at most $D$. Similarly to the previous analysis, the lowest ID agent could have taken at most $D$ steps during that time interval (using $D$ messages), the second lowest $\frac{D}{2}$, etc. The total number of messages used by the algorithm is, thus, at most $2 D+2 m+4 m=O(m)$.

Since the above algorithm is just a composition of well known techniques, we omit its formal description (and a more formal analysis). From the description and the discussion above, the theorem below follows easily. 
Theorem 4.1. There is a deterministic leader election algorithm that uses $O(m)$ messages.

\subsection{Matching both lower bounds simultaneously}

We first consider a leader election algorithms based on the least element (Least-El) list data structure, which was introduced in [7]. We first describe the Least-El data structure which assumes that each node $u$ is equipped with a $\operatorname{rank} \rho(u)$. Let $\operatorname{DIST}(u, v)$ denote the length of the shortest path between $u$ and $v$ in the input graph $G$. Given a set of nodes $S$, we say that $w \in S$ is the least element of $S$ if $\rho(w)<\rho(v)$, for all $v \in S$. For a node $u$, the least element list $\operatorname{LE}_{u}=\left\langle u, v_{1}, \ldots, v_{t}\right\rangle$ of $u$ is a list of nodes, such that, for any $v_{i} \in \mathrm{LE}_{u}$, it holds that $v_{i}$ is the least element of the $\operatorname{DIST}\left(u, v_{i}\right)$-neighborhood of $u$. By definition, the first entry of $\mathrm{LE}_{u}$ is $u$ itself, followed by $u$ 's smallest rank neighbor $v_{1}$ if $\rho\left(v_{1}\right)<\rho(u)$. The 3rd entry might contain $u$ 's 2-hop neighbor $v_{2}$ of smallest rank if $\rho\left(v_{2}\right)<\rho\left(v_{1}\right)$, and so forth.

For completeness, we now describe the distributed least element list algorithm of [11]. Each node $u$ chooses its rank $\rho(u)$ uniformly at random from the range $\left[1, n^{4}\right]$ and forwards $\rho(u)$ to its neighbors. Upon receiving $u^{\prime} s$ message, the neighbor $v$ adds an entry for $u$ to $\mathrm{LE}_{v}$ if $u$ 's rank is strictly smaller than $\rho(v)$. Otherwise, $v$ sends an echo message back to $u$. Nodes forward each newly added entry of their respective Least-El list once and discard any other received rank information. Note that every node $u$ receives all rank messages from distance $r$ in round $r$ and hence adds at most 1 entry to $\mathrm{LE}_{u}$ per round. For each ignored distance $r$ message, node $u$ sends an echo message that is forwarded to the respective distance $r$-neighbor. It follows that no new entries are added to $\mathrm{LE}_{u}$ after $D$ rounds, and after $O(D)$ rounds, all echo messages have reached their origin and each node knows that the Least-El list construction is complete, since they have received all echo messages from all neighbors. Now consider the (unique w.h.p.) node $v_{*}$ that has the smallest rank in the network. Clearly, $v_{*} \in \mathrm{LE}_{u}$, for every node $u$, and $v_{*}$ never adds any other entry to its own Least-El list. Thus $v_{*}$ can elect itself as the leader and every node knows that $v_{*}$ is the leader after $O(D)$ rounds. It is shown in [11] that $\left|\mathrm{LE}_{u}\right| \in O(\log n)$, for every node $u$ w.h.p., which implies that the total number of messages sent for constructing the Least-El lists is $O(m \log n)$. This yields a universal leader election algorithm that runs in $O(D)$ time, w.h.p., uses $O(m \log n)$ messages, and succeeds with high probability.

We now show that the least-element list algorithm when combined with a sparsification procedure yields optimal message and time complexity in sufficiently dense graphs. The distributed spanner construction of [6] yields a $k$-spanner $G^{\prime}$ with $n^{1+1 / k}$ edges in $O\left(k^{2}\right)$ rounds while using $O(\mathrm{~km})$ messages in the CONGEST model. Consider a fixed constant $\varepsilon>0$, we can obtain a $n^{1+\varepsilon / 2}$ spanner $G^{\prime}$ in $O(1)$ rounds using only $O(m)$ messages. Moreover, even if the graph is sparse, i.e. $m<n^{1+\varepsilon / 2}$, the spanner construction uses $O(m)$ messages and therefore does not worsen the total message complexity. Applying the algorithm of [11] to the sparsified graph $G^{\prime}$ shows that we can match both of our lower bounds in sufficiently dense graphs:

Corollary 4.2. Consider a network of $n$ nodes, $m$ edges, and diameter $D$ and assume that each node knows $n$. Let $\varepsilon>0$ be a fixed constant. If $m \geqslant n^{1+\varepsilon}$, then there is a randomized algorithm that achieves leader election with high probability, takes $O(D)$ time and has an expected message complexity of $O(m)$.

We now present Monte Carlo variants of the Least-El list election algorithm that (1) reduce the message complexity and (2) do not require any knowledge of $n$. Also, if the diameter $D$ is common knowledge, then we can get corresponding Las Vegas algorithms (cf. Cor. 4.6) 
Initially, after having sampled a random rank, each node becomes a candidate with probability $f(n) / n$, for a fixed $f(n) \leqslant n$ where $f(n) \in \Omega(1)$. (For now, we assume that nodes have knowledge of $n$. We will show below how to get rid of this assumption in the case of $f(n)=n$, i.e., when each node becomes candidate.) Each candidate $u$ chooses a random rank $\rho(u)$ from the range $\left[1, n^{4}\right]$ whereas non-candidate nodes set their rank to $n^{4}+1$. Only candidate nodes generate messages containing their own rank, while non-candidate nodes only update their own Least-El lists (not containing their own id) with received rank messages and forward these messages accordingly. By the above description, this ensures that the candidate with the smallest chosen rank becomes the leader after $O(D)$ rounds.

Lemma 4.3. The expected size of the Least-El list at each node is bounded by $O(\min (\log f(n), D))$.

Proof. Consider a node $v$ and define $N_{k}$ to be an ordering of the candidates within the $k$-neighborhood of $v$ that is non-decreasing w.r.t. distance to $v$. That is, the 2 nd entries of $N_{k}$ are candidates that are neighbors of $v$, followed by the 2-hop neighbors that became candidate and so forth, up to and including the distance $k$ candidates. Since the expected number of nodes that become candidate is $f(n)$, we also have $\mathbb{E}\left[\left|N_{k}\right|\right] \in f(n)$, for any $k$. Let $\left\langle u_{i}\right\rangle$ be the $i$-th (candidate) entry of $N_{k}$. We now consider the list $S_{k}$ that contains entry $\left\langle u_{i}\right\rangle$ iff $\rho\left(u_{i}\right)<\rho\left(u_{j}\right)$, for all $j<i$. Since ranks of candidates are chosen uniformly at random, the $i$-th entry is in $S_{k}$ with probability $\leqslant 1 / i$. This implies that $\mathbb{E}\left[\left|S_{k}\right|\right] \leqslant \sum_{i=1}^{\left|N_{k}\right|} \frac{1}{i} \in O(\log f(n))$. Observe that the probability of an entry to be in the least element list of $v$ (after $k$ rounds) is not larger than being in $S_{k}$. In other words, the size of list $S_{k}$ stochastically dominates the size of the $k$-round least element list at $v$, which implies $\mathbb{E}\left[\left|\mathrm{LE}_{v}\right|\right] \in O(\log f(n))$. Finally, we notice that the Least-El list of $v$ contains at most 1 entry for each distance $k(1 \leqslant k \leqslant D)$ and thus at most $D$ entries in total.

Theorem 4.4. Consider a network of $n$ nodes, $m$ edges, and diameter $D$ and assume that each node knows $n$. Let $f(n) \leqslant n$ be any function such that $f(n) \in \Omega(1)$. There is a randomized leader election algorithm $A$ that terminates in $O(D)$ rounds, has an expected message complexity of $O(m \cdot \min (\log f(n), D))$ and succeeds with probability $1-1 / e^{\Theta(f(n))}$. This implies the following:

(A) There is an algorithm that takes $O(D)$ time, has an expected message complexity of $O(\mathrm{~m}$. $\min (\log \log n, D))$ and succeeds with high probability.

(B) For any small constant $\varepsilon>0$, there is an algorithm that takes $O(D)$ time, sends $O(m)$ messages and succeeds with probability at least $1-\varepsilon$.

Proof. The algorithm succeeds if there is at least 1 candidate. Since nodes choose to become candidates independently and the expected number of candidates is $f(n)$, a standard Chernoff bound shows that the probability of having at least 1 candidate is $\geqslant 1-1 / e^{\Theta(f(n))}$ and, if $f(n) \in$ $\Theta(\log n)$, there is a candidate with high probability.

The termination time of the least element list construction is $O(D)$, which implies the sought bound on the time complexity. Recall that each node forwards each of its least element list entries exactly once to its neighbors. By Lemma 4.3, the expected size of each least element list is $O(\min (\log f(n), D))$. Note that a node sends each one of its (at most $\log f(n))$ list entries exactly once to each neighbor. Thus, the total number of rank messages sent by all (non-candidate and candidate) nodes is $O(m \cdot \min (\log f(n), D))$. Since nodes only send echo messages in response to received rank messages, the expected total number of message is $O(m \cdot \min (\log f(n), D))$ as required. Setting $f(n)=\Theta(\log n)$ proves (A). For (B), we consider any given $\varepsilon>0$ and set $f(n)=4 \log (1 / \varepsilon)$, which yields a message complexity of $O(m)$ and success with probability at least $1-\varepsilon$. 
Corollary 4.5. Consider a network of $n$ nodes, $m$ edges, and diameter $D$, and assume that nodes do not have knowledge of $n$. There is a randomized algorithm that achieves leader election with probability 1, takes $O(D)$ time and has a message complexity of $O(m \min (\log n, D)$ ) with high probability.

Proof. Consider the following protocol for estimating the network size $n$ : Each node $u$ flips an unbiased coin until the outcome is heads; let $X_{u}$ denote the random variable that contain the number of times that $X_{u}$ is flipped. Then, nodes exchange their respective values of $X_{u}$ whereas each node only forwards the highest value of $X_{u}$ (once) that it has seen so far. We observe that $X_{u}$ is geometrically distributed and denote its global maximum by $\bar{X}$. For any $u, \mathbb{P}\left[X_{u} \geqslant 2 \log _{2} n\right]=$ $1 / 2^{2 \log _{2} n}$, and by taking a union bound, $\mathbb{P}\left[\bar{X} \geqslant 2 \log _{2} n\right] \leqslant 1 / n$. Furthermore,

$$
\mathbb{P}\left[\bar{X}<\log _{2} n-\log _{2}(\log n)\right]=\left(1-1 / 2^{\log _{2} n-\log _{2}(\log n)}\right)^{n} \approx 1-\exp \left(-n / 2^{\log _{2} n-\log _{2}(\log n)}\right) .
$$

It follows that each node forwards at most $O(\log n)$ distinct values (w.h.p.) and thus the number of messages is $O(m \log n)$. To detect termination, we use the same echo mechanism as in the least element list algorithm described above. After $O(D)$ rounds, each node knows the value of $\bar{X}$, denoted by $x$, and sets its estimate $\hat{n}=2^{x}$. Due to the above bounds on $\bar{X}$ it follows that (w.h.p.) $\hat{n} \in O\left(n^{2}\right)$ and $\hat{n} \in \Omega(n / \log n)$.

Then, nodes execute the least element list algorithm with some crucial differences: First, we note that since with high probability all ranks are unique, applying Theorem 4.4 yields the same bounds as in the case where $n$ is known exactly. Moreover, nodes use $\hat{n}$ and $f(n)=\hat{n}$ when running the least element list algorithm, i.e., every node becomes candidate. We include the (preassigned) unique node ids when comparing ranks to break ties. This guarantees that there is always a unique node with highest (rank,ID) pair and thus there is exactly 1 leader.

Finally, if nodes know both $n$ and $D$, we can transform the Monte Carlo algorithm of Theorem 4.4 to a Las Vegas algorithm, by instructing nodes to restart the algorithm if no messages were received during $\Theta(D)$ rounds and repeat until eventually a leader is elected. In the case $f(n) \in \Theta(1)$, there is a constant probability of having at least 1 candidate. Thus the expected number of times that nodes need to restart is also constant, and each rerun of the algorithm takes $O(D)$ time and has an expected $O(m)$ message complexity.

Corollary 4.6. Consider a network of $n$ nodes, $m$ edges, and diameter $D$, and assume that nodes have knowledge of $n$ and $D$. There is a randomized algorithm that achieves leader election with probability 1 , has an expected time complexity of $O(D)$ and an expected message complexity of $O(m)$.

\subsection{Approaching the lower bounds}

Approaching by a randomized algorithm: We now present an algorithm that first sparsifies the given network and then solves leader election by instantiating the algorithm of Theorem 4.4. In contrast to the sparsification procedure used for Corollary 4.2, the bounds of our clustering algorithm hold in all graphs. In more detail ${ }^{3}$, our algorithm proceeds in 3 phases. We first randomly select $\Theta(\log n)$ candidates and each candidate starts constructing a BFS tree in Phase 1. At the end of this phase the network is partitioned into $\Theta(\log n)$ clusters and each cluster consists

\footnotetext{
${ }^{3}$ See Algorithm 1 for the complete pseudo code.
} 
of only $O(n)$ tree edges. Note that we can construct the BFS trees in $O(D)$ time by sending $O(m)$ messages.

In Phase 2, we sparsify the inter-cluster edges. The crucial observation is that, for maintaining a diameter of $O(D \log n)$ we can discard all but 1 inter-cluster edge for each pair of adjacent clusters. This sparsification process is initiated locally by the BFS nodes that are adjacent to nodes in distinct BFS trees. Each such node locally sparsifies the inter-cluster edges and then propagates the updated inter-cluster graph to its BFS parent. Since there are $\Theta(\log n)$ clusters with high probability, the size of this (sparsified) inter-cluster graph is bounded by $O\left(\log ^{2} n\right)$, and can hence be sent across an edge in $O(\log n)$ rounds. Thus it takes $O(D \log n)$ time (w.h.p.) until each root has computed the final inter-cluster graph, which in turn is propagated downwards through the tree. This ensures that every BFS node knows the same inter-cluster graph by the end of Phase 2 .

After sparsifying, we are left with $O\left(n+\log ^{2} n\right)$ edges in total. In Phase 3, we execute the algorithm of Theorem 4.4 on this network, requiring only $O(n \log n)$ additional messages and $O(D \log n)$ time. Overall, the message complexity is $O(m+n \log n)$, whereas the time is dominating by $O(D \log n)$, with high probability.

Theorem 4.7. Consider any network of $n$ nodes, $m$ edges, and diameter $D$, and assume that each node has knowledge of $n$. With high probability, Algorithm 1 elects a unique leader in $O(D \log n)$ rounds and uses $O(m+n \log n)$ messages.

Proof. Let $X$ be the random variable representing the number of candidates. The expected number of candidates is $8 \log n$. By invoking a standard Chernoff bound (cf. Theorems 4.4 and 4.5 in [17]), we get that there are $\Theta(\log n)$ candidates (and therefore $\Theta(\log n)$ distinct clusters) with high probability.

We now argue the message complexity bound for Phase 1: By the description of the algorithm, each node $v$ sends at most one join request, either due to $v$ being a candidate, or triggered by receiving a (first) join request from a neighbor. Since $v$ only responds to the first join request, $v$ sends at most $1\langle$ ack $\rangle$ message. It follows that every node sends a constant number of messages of $O(\log n)$ size over each incident link and hence the total number of messages sent in Phase 1 is $O(m)$.

To bound the message complexity of Phase 2, we need to argue that any message containing graph INTER-GRAPH is of size $O\left(\log ^{2} n\right)$ : Note that a leaf node $w$ first sparsifies INTER-GRAPH $w$ according to Line 13, before sending it to its parent. With high probability, there are no more than $O(\log n)$ clusters, which tells us that the number of distinct edge labels in INTER-GRAPH ${ }_{w}$ is bounded by $O(\log n)$. Since isolated vertices are removed from INTER-GRAPH ${ }_{w}$ before forwarding, we get that $\mid$ INTER-GRAPH $w \mid \in O\left(\log ^{2} n\right)$. For any non-leaf node $v$, it is sufficient to observe that node $v$ performs the same sparsification procedure like leaf nodes after merging the graphs received from $v$ 's children. Thus, by the above argument, this again results in INTER-GRAPH $v \in O\left(\log ^{2} n\right)$, as required. Finally, when the candidate (root) node $u$ initiates the broadcasting (cf. Line 15) of INTER-GRAPH ${ }_{u}$ (of $O\left(\log ^{2} n\right)$ size) to its children who simply forward the (unchanged) graph INTER-GRAPH ${ }_{u}$ along the edges of the BFS tree. Note that all communication in Phase 2 uses only the $O(n)$ BFS tree edges and, in particular, no messages are sent across inter-cluster edges. Since nodes can send messages of size $O(\log n)$ and each node sends INTER-GRAPH over a tree edge at most twice, the total number of messages in Phase 2 is $O(n \log n)$ with high probability.

After Phase 2, the sparsified network has $O\left(n+\log ^{2} n\right)=O(n)$ edges (w.h.p.), which, according to Theorem 4.4, bounds the additional messages of invoking the algorithm of Theorem 4.4 in Phase 3 
$\overline{\text { Algorithm } 1 \text { The Clustering Algorithm }}$

\section{Phase 1: Cluster Construction}

2: Overview: Each candidate $u$ constructs a BFS tree and every non-candidate $w$ joins one of these BFS trees. After Phase 1, each node $v$ maintains 2 graphs: 1. TREE $v$ contains the (local) neighborhood of the BFS tree that $v$ has joined. 2. INTER-GRAPH $v$ represents the connections to neighbors of $v$ that joined different BFS trees.

3: for each candidate $u$ (in parallel) do

4: $\quad$ Node $u$ sets CLUSTER $_{u} \leftarrow u$ and initiates the construction of a BFS tree by forwarding a $\langle j o i n, u\rangle$ request to all neighbors.

5: Tree edges: If a non-candidate node $w$ receives a join request $\langle j o i n, u\rangle$ for the first time, it sets CLUSTER $_{w} \leftarrow u$ and forwards $\langle$ join, $u\rangle$ to all its neighbors except to node $v$ that sent the request. Then, node $w$ sends an 〈ack $\rangle$ message to $v$. We call $v$ the parent of $w$ and $w$ a child of $v$. (If $w$ receives multiple join requests simultaneously the first time, it arbitrarily picks one request and discards all others.) After $w$ has received the first join request in some round, it ignores any subsequently arriving join requests.

6: $\quad$ Each node $w$ adds the edges to its parent and its children to $\operatorname{TREE}_{w}$.

7: $\quad$ Inter-cluster edges: For each neighbor $v \notin \operatorname{TREE}_{w}$, node $w$ adds the edge $(w, v)$ that is labeled with CLUSTER $_{v}$ to the graph INTER-GRAPH $w$.

8: After Phase 1, only edges in TREE and INTER-GRAPH are used for communication and each node $v$ belongs to some BFS tree determined by $\operatorname{CLUSTER}_{v}$.

Phase 2: Sparsify Inter-Cluster Edges

9: Overview: In this phase, we reduce the inter-cluster edges to $O\left(\log ^{2} n\right)$ in total, by retaining no more than 1 connection between any pair of clusters.

10: for each BFS tree $T$ (in parallel) do

11: $\quad$ Every leaf node $w$ in $T$ performs sparsification on its graph INTER-GRAPH I $_{w}$ as described in Line 13 and then forwards INTER-GRAPH $w$ to its parent.

12: Merging: Every (non-leaf) node $v$ waits until it has received INTER-GRAPH from all of its children. Let INTER-GRAPH $w_{1}, \ldots$, INTER-GRAPH $_{w_{j}}$ be the received graphs. Node $v$ adds all edges and (distinct) vertices of these graphs (if any) to its own graph INTER-GRAPH $v$.

13: Sparsify: Let $C$ be the distinct edge labels (i.e. cluster ids) in INTER-GRAPH $v$ of node $v$. For each cluster id $c_{i} \in C$, node $v$ discards all but one edge with label $c_{i}$ from INTER-GRAPH $v$, i.e., $\mid E\left(\right.$ INTER-GRAPH $\left._{v}\right)|=| C \mid$ after this step; node $v$ also removes any isolated vertices.

14: Node $v$ forwards INTER-GRAPH $v$ to its (BFS tree) parent. (This might take multiple rounds.)

15: Once the candidate (root) node $u$ has merged all graphs from its children, it broadcasts the updated graph INTER-GRAPH ${ }_{u}$ to all children. (This might take multiple rounds.)

16: $\quad$ Upon receiving INTER-GRAPH ${ }_{u}$ from a parent $u$, node $v$ sets its own graph to

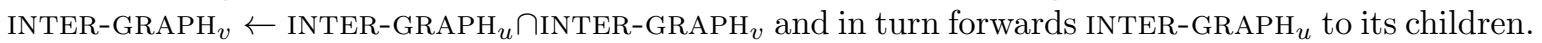

Phase 3: Perform Election:

17: Invoke the algorithm of Theorem 4.4 (with $f(n)=n$ ) on the network $\left(\bigcup_{v} \operatorname{TREE}_{v}\right) \cup\left(\bigcup_{v}\right.$ INTER-GRAPH $\left._{v}\right)$.

by $O(n \log n)$. Summing up over Phases 1-3, we get a total message complexity of $O(m+n \log n)$ with high probability.

Observe that Phases 1-2 construct an overlay network that is connected, since the sparsification phase retains one inter-cluster edge for any two clusters that are adjacent in the original network. Thus, we run the algorithm of Theorem 4.4 in Phase 3 on a connected network. Since we have 
$\Theta(\log n)$ candidates with high probability, the correctness of electing exactly 1 leader follows from Theorem 4.4.

Next, we analyze the time complexity. Clearly, the time for constructing a BFS tree is bounded by $O(D)$. Moreover, constructing INTER-GRAPH $w$ at any node $w$ does not require additional time. Hence the total number of rounds of Phase 1 is bounded by $O(D)$. During Phase 2, nodes communicate only with nodes in the same BFS tree. According to our argument for the message complexity, at most $O(\log n)$ messages are sent over each tree edge during Phase 2, since INTER-GRAPH is sent at most twice by each node. In the worst case, this induces a congestion of $O(\log n)$ at each affected (tree) edge. Thus we can bound the total number of rounds to complete Phase 2 by $O(D \log n)$. In Phase 3, we invoke the algorithm of Theorem 4.4, takes $O\left(D^{\prime}\right)$ rounds on any network with diameter $D^{\prime}$. Since the diameter of each BFS tree is at most $O(D)$ and we have $\Theta(\log n)$ BFS trees (w.h.p.), it follows that the diameter of the sparsified network (on which we execute the algorithm of Theorem 4.4) is bounded by $O(D \log n)$. This implies that Algorithm 1 terminates in $O(D \log n)$ rounds with high probability.

Approaching the bounds deterministically: We now present a deterministic algorithm cf. Algorithm 2 that can achieve Leader Election in $O(D \log n)$ time and $O(m \log n)$ messages, where $D$ is the diameter, $n$ the number of nodes and $m$ the number of edges of the graph. This algorithm works without any knowledge of parameters $D, n$ or $m$. We show later that there is a somewhat simpler algorithm that achieves the same bounds if $D$ is known to the nodes.

Informally, in Algorithm 2, all nodes wakeup simultaneously in phase 1 and consider themselves to be Leader candidates. In each phase $p$, every candidate $v$ in that phase tries to grow a BFS tree (kingdom) of radius $2^{p}$ (i.e. every node within distance $2^{p}$ of $v$ considers $v$ as their leader candidate). However, messages from different candidates can now collide at inbetween nodes and an election ensues. In an election between two nodes, the node in the higher phase (or higher ID, if they have the same phase) wins. In fact, the higher phase node just continues growing its kingdom as if no collision happened. We call the process in each phase the 4-stage Election. The messages in the stages are called ELECT, ACK, CONFIRM and VICTOR. The ACK messages tell a candidate if it has succesfully grown its kingdom without collisions or if it has won all its collisions. If it lost, it also comes to know the identity of the highest winner. Using CONFIRM messages, it broadcasts this identity to its neighboring candidates. As before, the CONFIRM messages collide and the information about the highest winner is convergecast back using VICTOR messages. Consider the graph of candidates at this point, where each node is a kingdom and an edge exists between two kingdoms if and only if they collide. The 4-stage election implies that candidates know not only about their neighbors but also about their neighbor of neighbors. Thus, a candidate that wins its phase wins over not only its neighbors but also the neighbor of neighbors. As soon as a candidate wins a phase, it proceeds to the next phase. As the number of candidates exponentially decreases, this ensures that the algorithm terminates in $O(\log n)$ time (Lemma 4.8). Moreover, an edge is used at most a constant number of times in a phase giving a message complexity of $O(m \log n)$ (Lemma 4.9).

As mentioned before, candidates maybe in different phases in the algorithm at a given time. However, candidates in the same phase may also be at different stages. In particular, an ELECT message from one candidate may collide with messages at later stage of another candidate (at least one of such colliding messages must be an ELECT message). If a candidate is late for all its elections with another node, the candidate sets its state to nonelected but continues the present phase as usual. Our main result is stated in Theorem 4.10. 
Algorithm 2 The Double-Win Growing Kingdom Algorithm. Candidates try to conquer exponentially larger territories in each phase, but if they get defeated by a higher ID candidate, they also inform their neighboring candidates about this higher ID candidate. A leader is elected after $O(D \log n)$ phases.

1: Algorithm proceeds in phases $p=1,2, \ldots$ Within each phase, there are 4 stages (each with its particular type of message). If messages from different candidates reach the same node, we say that a collision has occurred. Messages from a higher phase ignore collisions with messages of a lower phase and proceed as if the collision did not occur (i.e. it overruns). If messages of the same phase collide, higher stage messages win over lower stages, and for messages of the same stage, the higher ID message wins. The format of an $A C K()$ message is ACK(Origin, VALUE, LATEFLAG) where Origin is the node where the message originated (e.g. where a collision occured), VALUE is the value used for election (e.g. IDs), and LATEFLAG is a flag set to LATE if the collision involved messages of different phases and set to OK otherwise.

2: for all candidate nodes $v$ (in parallel) in phase $p$ do 4-stage Election:

3: $\quad$ (Stage 1) Phase $p$, Step 1: Node $v$ initiates the construction of a BFS tree of depth $2^{p-1}$ by sending ELECT(phase,v, Counter) message to all neighbors, where Counter is set to $2^{p-1}$.

4: $\quad$ if $E L E C T\left(w^{\prime} . p h a s e, w^{\prime} . I D\right.$, Counter $)$ message received from neighbors $w^{\prime}$, where $w . I D$ is the highest ID seen (including the $I D$ of $v$ ) then

5: $\quad$ (Stage 2) Set $v \cdot \operatorname{Max}=(w \cdot p h a s e, w \cdot I D)$. Send $A C K(v, v \cdot M a x, O K)$ to $w^{\prime}$

6: $\quad$ (Stage 3) $v$ receives $A C K\left(w, v_{i} . M a x, L A T E F L A G\right)$ from all its neighbors $i$ (where $v_{i} . I D$ may even be $v . I D$ (if $v$ won)) Set $v . M a x$ to the highest $\left(v_{i}\right.$. phase $\left._{i}, v_{i} . I D\right)$. Broadcast CONFIRM(v.Max) along $v$ 's BFS tree.

7: $\quad$ if there exists a $w$, all of the $A C K()$ messages from which have their LATEFLAG set then $\{v$ was late for an election\}

8: $\quad$ set state to NON-ELECTED(non-candidate).

9: $\quad$ (Stage 4) $v$ receives $\operatorname{VICTOR}\left(v_{i}\right.$.phase, $v_{i} . I D$ ) from all its neighbors $i$. If $v$. Max is the highest (of $\left(v_{i}\right.$.phase, $\left.v_{i} . I D\right)$ ), then, if $v$ is not in state NON-ELECTED, $v$ continues as a candidate for the next round else sets its state to NON-ELECTED(non-candidate).

10: $\quad$ for non-candidate node $z$ do

11: $\quad$ (No Collision) If $z$ is already part of $v$ 's BFS tree (from the previous phase) and receives only the message Elect(v.phase, v.ID, counter) message from $z$ 's parent, decrement counter and forward $v$ 's Elect() message to its children.

\section{Collision and 4-Stage Election:}

12: $\quad$ If $v$ 's Elect() message reaches $z$ and $v$ has a higher phase than $z . M a x$, then $z$ becomes part of $v$ 's BFS tree for this (higher) phase (If it received $z$ 's message from multiple nodes simultaneously, it selects one of them arbitrarily as its parent and sends an ack to the rest), decrements the counter of the message and forwards $z$ 's message to nodes it has not heard from in this phase (marking them as children in the BFS tree). $v$ then awaits $A C K$ messages from its children to convergecast back.

13: $\quad$ (Stage 1)If multiple Elect() messages of the same phase collide at $z$ and $v$ has the highest $I D$ of them, $z$ sets $z . M a x$ to $v$ 's fields, and broadcasts $v$ 's Elect() message further to neighbors $z$ hasn't talked to in this phase and awaits their $A C K()$. If $z$ has no such neighbors, it enters Stage 2 and convergecasts back $A C K(z \cdot \operatorname{Max})$

14: (Stage 2) Convergecast $A C K(z . M a x)$ to $z$ 's parent. If $z$ received an Elect(v) message of the same phase as Max, an $A C K(z, z \cdot M a x, L A T E)$ message is convergecast back to $v$.

15: $\quad$ (Stage 3) Broadcast CONFIRM(z.Max) to $z$ 's children in $v$ 's BFS tree.

16: $\quad$ (Stage 4) Convergecast $\operatorname{VICTOR}(z . M a x)$ to $z$ 's parent.

17: Termination: During the BFS growth phase (Stage 1), each node detects if it is the leaf of the fully grown tree (i.e. it is a leaf and its distance from the root is less than $2^{p}$ for phase $p$ ) and convergecasts this information back. If all the neighbors of candidate $v$ convergecast this information, then $v$ knows it is the only candidate left (and becomes leader). 
First, we prove the following lemmas about Algorithm 2 on a network of $n$ nodes, $m$ edges, and diameter $D$.

Lemma 4.8. There are at most $\frac{n}{2^{p-1}}$ candidates at any point in time during phase $p$.

Proof. The proof is by induction on $p$. For the base case, consider $p=1$. All the $n$ nodes are candidates at step 1 of phase 1 . This phase lasts 4 steps. Assume that more than $n / 2$ candidates remain at the end of this phase (i.e. step 4). We will show that this yields a contradiction: By the description of the algorithm, each node gets the IDs of its neighboring nodes and then informs each of them of the highest ID it saw. Consider two surviving candidates $v$ and $w$. Nodes $v$ and $w$ must be separated by at least two nodes, else their IDs would have reached each other (possibly via a common neighbor). Let us say a node $z$ dominates node $x$ at time $t$ if $x$ got a message originating from $z$ at some time $t^{\prime} \leqslant t$ and at time $t$, node $z$ survives but $x$ does not. Clearly, $v$ and $w$ dominate at least one node each, hence, the number of surviving candidates cannot be more than $n / 2$ and all of them proceed to phase 2 at the beginning of the next step.

By the inductive hypothesis, there are at most $\frac{n}{2^{p-2}}$ candidates in phase $p-1$. If there are at most $\frac{n}{2^{p-1}}$ such candidates, we are done. Each candidate in phase $p$ tries to grow to a radius of $2^{p-1}$. If each of them succeeds, this implies that there are at most $\frac{n}{2^{p-1}}$ such candidates. (Consider a path composed of the longest paths of these kingdoms; this path cannot exceed the diameter, which is bounded by $n-1)$. Thus we consider the case when there are collisions. There were at most $\frac{n}{2^{p-2}}$ candidates of phase $p-1$ to begin with, and each of them tries to grow to a radius of $2^{p-1}$. Consider the graph where each of these kingdoms is a node (label the node by its candidate) and an edge exists between two nodes if and only if they suffer a collision. By a similar argument as above (each node collects the maximum ID of all its neighbors and informs them of the maximum ID), each pair of the surviving colliding candidates is 3-separated (has at least two other nodes inbetween) in this graph if there is a path between them. Thus, each candidate dominates at least one other (defeated) candidate and the number of surviving candidates reaching phase $p$ is at most $\frac{n}{2^{p-1}}$.

Lemma 4.9. Each phase of Algorithm 2 uses $O(m)$ messages.

Proof. Consider an edge $(x, y)$ and count the number of messages of a particular phase $p$ that go over it. By the description of the algorithm, the election happens in four different stages (each stage uses a different kind of message). If $(x, y)$ belonges to node $v$ 's BFS tree in phase $p-1$, only one message for each stage traverses this edge. Only if the edge belongs to the border of two colliding kingdoms, then messages from both the colliding kingdoms may traverse the edge, but this only doubles the number of messages. Thus, phase $p$ takes $O(m)$ messages in total.

Theorem 4.10. Consider any network of $n$ nodes, $m$ edges, and diameter $D$. There is a deterministic algorithm that elects a unique leader in $O(D \log n)$ time while using $O(m \log n)$ messages.

Proof. According to Lemma 4.8, there can be at most one node in phase $\log n+1$. A message from a candidate can travel at most distance $D$ (in each stage of the election) in any phase and all messages in the same phase travel in parallel without congestion. It is possible that messages from two different candidates (which are in two different phases) traverse an edge but these messages can be piggybacked and only double the number of messages. Further, as soon as a phase is completed, if a candidate survives one phase, it proceeds to the next. Thus, each stage can take at most $4 D$ time for completion. At the end of at most $4 D(\log n)$ steps, we are left with only one node and 
this node enters phase $\log n+1$. Using a simple termination condition(Algorithm 2: line 17), this node can detect that it is the only candidate left and declare itself the leader. Since there are only $\log n+1$ phases, from Lemma 4.9, we get that the algorithm uses $O(m \log n)$ messages.

\section{Knowledge of $D$}

If the nodes knew the diameter $D$, Algorithm 2 could be simplified in the following way. The algorithm will now consist of (at most $\log n$ ) phases. Each phase consists of exactly $4 D$ time steps. All nodes wake up simultaneously at the beginning of the algorithm and try to grow their kingdom to a radius of $D$. In case of collision, the algorithm proceeds as in Algorithm 2 except that now all the candidates are in the same phase and same stages. If a candidate finishes its task for the current phase sooner than in $4 D$ time, it waits till the end of that phase. Now, all candidates that move to the next phase start the next phase simultaneously. With an analysis similar to that of Lemma 4.8, it follows that the number of surviving candidates (at least) halves in each phase. Thus, this simplified algorithm terminates with a leader in $O(D \log n)$ time and $O(m \log n)$ message. Algorithm 2 could be similarly modified so that all candidates of a phase $p$ start simultaneously and try to grow their kingdom to $2^{p}$. However, if the nodes do not know $D$ and there are $O(\log n)$ phases, it is possible that the larger phases take $O(n)$ steps. This is because some nodes may

now have to wait for $2^{O(\log n)}$ steps after having collided with other candidates much earlier in the phase.

\section{Conclusion}

We studied the role played by randomization in universal leader election. Some open questions on universal leader election raised by our work are: Is there a deterministic leader election algorithm that runs in $O(D)$ rounds and uses only $O(m+n \log n)$ messages in the worst case? Can we find tight (universal) upper and lower bounds for general graphs with and without knowledge of $n$ ? What is the precise trade-off between the probability of success and simultaneously approaching the $\Omega(D)$ and $\Omega(m)$ lower bounds?

\section{Acknowledgements}

We thank the anonymous PODC 2013 reviewers for helpful comments and the PODC program committee for inviting the paper to the Journal of the ACM.

\section{References}

[1] Hosame Abu-Amara and Arkady Kanevsky. On the complexities of leader election algorithms. In Proceedings of the Fifth International Conference on Computing and Information (ICCI), pages $202-206$, may 1993.

[2] Yehuda Afek and Eli Gafni. Time and message bounds for election in synchronous and asynchronous complete networks. SIAM Journal on Computing, 20(2):376-394, 1991.

[3] Hagit Attiya and Jennifer Welch. Distributed Computing: Fundamentals, Simulations and Advanced Topics (2nd edition). John Wiley Interscience, March 2004. 
[4] Baruch Awerbuch. Optimal distributed algorithms for minimum weight spanning tree, counting, leader election, and related problems. In Proceedings of the nineteenth annual ACM symposium on Theory of computing, STOC '87, pages 230-240, New York, NY, USA, 1987. ACM.

[5] Baruch Awerbuch, Oded Goldreich, Ronen Vainish, and David Peleg. A trade-off between information and communication in broadcast protocols. J. ACM, 37(2):238-256, April 1990.

[6] Surender Baswana and Sandeep Sen. A simple and linear time randomized algorithm for computing sparse spanners in weighted graphs. Random Struct. Algorithms, 30(4):532-563, 2007.

[7] Edith Cohen. Size-estimation framework with applications to transitive closure and reachability. J. Comput. Syst. Sci., 55(3):441-453, 1997.

[8] Greg N. Frederickson and Nancy A. Lynch. Electing a leader in a synchronous ring. Journal of the ACM, 34(1):98-115, 1987.

[9] Emanuele G. Fusco and Andrzej Pelc. Knowledge, level of symmetry, and time of leader election. In ESA, pages 479-490, 2012.

[10] Saurabh Ganeriwal, Ram Kumar, and Mani B. Srivastava. Timing-sync protocol for sensor networks. In Proceedings of the 1st international conference on Embedded networked sensor systems, SenSys '03, pages 138-149, New York, NY, USA, 2003. ACM.

[11] Maleq Khan, Fabian Kuhn, Dahlia Malkhi, Gopal Pandurangan, and Kunal Talwar. Efficient distributed approximation algorithms via probabilistic tree embeddings. Distributed Computing, 25(3):189-205, 2012.

[12] Ephraim Korach, Shay Kutten, and Shlomo Moran. A modular technique for the design of efficient distributed leader finding algorithms. ACM Trans. Program. Lang. Syst, 12(1):84-101, 1990.

[13] Ephraim Korach, Shlomo Moran, and Shmuel Zaks. Optimal lower bounds for some distributed algorithms for a complete network of processors. Theoretical Computer Science, 64(1):125 $132,1989$.

[14] Shay Kutten, Gopal Pandurangan, David Peleg, Peter Robinson, and Amitabh Trehan. Sublinear bounds for randomized leader election. In ICDCN'13, pages 348-362, 2013.

[15] Gérard Le Lann. Distributed systems - towards a formal approach. In IFIP Congress, pages 155-160, 1977.

[16] Nancy Lynch. Distributed Algorithms. Morgan Kaufman Publishers, Inc., San Francisco, USA, 1996.

[17] M. Mitzenmacher and E. Upfal. Probability and Computing: Randomized Algorithms and Probabilistic Analysis. Cambridge University Press, 2004.

[18] Rajeev Motwani and Prabhakar Raghavan. Randomized Algorithms. Cambridge University Press, 1995. 
[19] Erik Nygren, Ramesh K. Sitaraman, and Jennifer Sun. The Akamai network: a platform for high-performance internet applications. SIGOPS Oper. Syst. Rev., 44(3):2-19, August 2010.

[20] David Peleg. Time-optimal leader election in general networks. Journal of Parallel and Distributed Computing, 8(1):96 - 99, 1990.

[21] David Peleg. Distributed Computing: A Locality-Sensitive Approach. SIAM, Philadelphia, 2000 .

[22] Nicola Santoro. Design and Analysis of Distributed Algorithms (Wiley Series on Parallel and Distributed Computing). Wiley-Interscience, 2006.

[23] Gerard Tel. Introduction to distributed algorithms. Cambridge University Press, New York, NY, USA, 1994.

[24] Yong Yao and Johannes Gehrke. The cougar approach to in-network query processing in sensor networks. SIGMOD Rec., 31(3):9-18, September 2002. 